\title{
Intrinsic sign problem in fermionic and bosonic chiral topological matter
}

\author{
Omri Golan $\odot,{ }^{1, *}$ Adam Smith $\odot,{ }^{2}$ and Zohar Ringel ${ }^{3}$ \\ ${ }^{1}$ Department of Condensed Matter Physics, Weizmann Institute of Science, Rehovot 76100, Israel \\ ${ }^{2}$ Department of Physics, TFK, Technische Universität München, James-Franck-Straßle 1, D-85748 Garching, Germany \\ ${ }^{3}$ Racah Institute of Physics, The Hebrew University of Jerusalem, Jerusalem 9190401, Israel
}

(Received 4 June 2020; revised 2 September 2020; accepted 3 September 2020; published 6 October 2020)

\begin{abstract}
The infamous sign problem leads to an exponential complexity in Monte Carlo simulations of generic many-body quantum systems. Nevertheless, many phases of matter are known to admit a sign-problem-free representative, allowing efficient simulations on classical computers. Motivated by long-standing open problems in many-body physics, as well as fundamental questions in quantum complexity, the possibility of intrinsic sign problems, where a phase of matter admits no sign-problem-free representative, was recently raised but remains largely unexplored. Here we establish the existence of an intrinsic sign problem in a broad class of gapped, chiral, topological phases of matter. Within this class, we exclude the possibility of stoquastic Hamiltonians for bosons (or "qudits") and of sign-problem-free determinantal Monte Carlo algorithms for fermions. The intrinsically sign-problematic class of phases we identify is defined in terms of topological invariants with clear observable signatures: the chiral central charge and the topological spins of anyons. We obtain analogous results for phases that are spontaneously chiral, and present evidence for an extension of our results that applies to both chiral and nonchiral topological matter.
\end{abstract}

DOI: 10.1103/PhysRevResearch.2.043032

\section{INTRODUCTION}

Utilizing a random sampling of phase-space according to the Boltzmann probability distribution, Monte Carlo simulations are arguably the most powerful tools for numerically evaluating thermal averages in classical many-body physics [1]. Though the phase-space of an $N$-body system scales exponentially with $N$, a Monte Carlo approximation with a fixed desired error is usually obtained in polynomial time [2,3]. In quantum Monte Carlo (QMC), one attempts to perform Monte Carlo computations of thermal averages in quantum manybody systems, by following the heuristic idea that quantum systems in $d$ dimensions are equivalent to classical systems in $d+1$ dimensions $[4,5]$.

The difficulty with any such quantum to classical mapping, henceforth referred to as a method, is the infamous sign problem, where the mapping can produce complex, rather than non-negative, Boltzmann weights $p$, which do not correspond to a probability distribution. Faced with a sign problem, one can try to change the method used and obtain $p \geqslant 0$, thus curing the sign problem [6,7]. Alternatively, one can perform QMC using the weights $|p|$, which is often done but generically leads to an exponential computational complexity

\footnotetext{
*omri.golan@weizmann.ac.il

Published by the American Physical Society under the terms of the Creative Commons Attribution 4.0 International license. Further distribution of this work must maintain attribution to the author(s) and the published article's title, journal citation, and DOI.
}

in evaluating physical observables, limiting one's ability to simulate large systems at low temperatures [2].

Conceptually, the sign problem can be understood as an obstruction to mapping quantum systems to classical systems, and accordingly, from a number of complexity theoretic perspectives, a generic curing algorithm in polynomial time is not believed to exist $[2,6-10]$. In many-body physics, however, one is mostly interested in universal phenomena, i.e., phases of matter and the transitions between them, and therefore representative Hamiltonians which are sign-free often suffice [11]. In fact, QMC simulations continue to produce unparalleled results, in all branches of many-body quantum physics, precisely because new sign-free models are constantly being discovered [5,11-17].

Designing sign-free models requires design principles (or "de-sign" principles) [11,18]—easily verifiable properties that, if satisfied by a Hamiltonian and method, lead to a signfree representation of the corresponding partition function. An important example is the condition $\langle i|H| j\rangle \leqslant 0$ where $i \neq j$ label a local basis, which implies non-negative weights $p$ in a wide range of methods $[10,11]$. Hamiltonians satisfying this condition in a given basis are known as stoquastic [8], and have proven very useful in both application and theory of QMC in bosonic (or spin, or "qudit") systems [2,6-11].

Fermionic Hamiltonians are not expected to be stoquastic in any local basis [2,5], and alternative methods, collectively known as determinantal quantum Monte Carlo (DQMC), are therefore used $[4,5,17,19,20]$. The search for design principles that apply to DQMC, and applications thereof, has naturally played the dominant role in tackling the sign problem in fermionic systems and has seen a lot of progress in recent years $[5,17,18,21-24]$. Nevertheless, long-standing 
TABLE I. Examples of intrinsic sign problems based on the criterion $e^{2 \pi i c / 24} \notin\left\{\theta_{a}\right\}$, in terms of the chiral central charge $c$ and the topological spins $\theta_{a}=e^{2 \pi i h_{a}}$. The number of spins $h_{a}$ is equal to the dimension of the ground-state subspace on the torus. We mark bosonic/fermionic phases by $(\mathrm{B} / \mathrm{F})$. The quantum Hall Laughlin phases correspond to $U(1)_{q}$ Chern-Simons theories. The $\ell$-wave superconductor is chiral, e.g., $p+i p$ for $\ell=1$, and comprising a single flavor of spin-less fermions. Data for the spin-full case are identical to that of the Chern insulator, with $-\ell$ odd (even) in place of $v$, for triplet (singlet) pairing. The modulo 8 ambiguity in the central charge of the Fibonacci anyon model corresponds to the stacking of a given realization with copies of the $E_{8} K$-matrix phase. Data for the three quantum Hall Pfaffian phases are given at the minimal filling $1 / 2$. The physical filling $5 / 2$ is obtained by stacking with a $v=2$ Chern insulator, and an intrinsic sign problem appears in this case as well. Relevant computations can be found in the Supplemental Material [68].

\begin{tabular}{|c|c|c|c|c|}
\hline Phase of matter & Parameterization & $c$ & $\left\{h_{a}\right\}$ & Intrinsic sign problem? \\
\hline Laughlin (B) [36] & Filling $1 / q,(q \in 2 \mathbb{N})$ & 1 & $\left\{a^{2} / 2 q\right\}_{a=0}^{q-1}$ & In $98.5 \%$ of first $10^{3}$ \\
\hline Laughlin (F) [36] & Filling $1 / q,(q \in 2 \mathbb{N}-1)$ & 1 & $\left\{(a+1 / 2)^{2} / 2 q\right\}_{a=0}^{q-1}$ & In $96.7 \%$ of first $10^{3}$ \\
\hline Chern insulator (F) [App. B] & Chern number $v \in \mathbb{Z}$ & $v$ & $\{v / 8\}$ & For $v \notin 12 \mathbb{Z}$ \\
\hline$\ell$-wave superconductor $(\mathrm{F})[61]$ & Pairing channel $\ell \in 2 \mathbb{Z}-1$ & $-\ell / 2$ & $\{-\ell / 16\}$ & Yes \\
\hline Kitaev spin liquid (B) [46] & Chern number $v \in 2 \mathbb{Z}-1$ & $v / 2$ & $\{0,1 / 2, v / 16\}$ & Yes \\
\hline SU $(2)_{k}$ Chern-Simons (B) [69] & Level $k \in \mathbb{N}$ & $3 k /(k+2)$ & $\{a(a+2) / 4(k+2)\}_{a=0}^{k}$ & In $91.6 \%$ of first $10^{3}$ \\
\hline$E_{8} K$-matrix (B) $[70]$ & Stack of $n \in \mathbb{N}$ copies & $8 n$ & $\{0\}$ & For $n \notin 3 \mathbb{N}$ \\
\hline Fibonacci anyon model (B) [69] & & $14 / 5(\bmod 8)$ & $\{0,2 / 5\}$ & Yes \\
\hline Pfaffian (F) [71] & & $3 / 2$ & $\{0,1 / 2,1 / 4,3 / 4,1 / 8,5 / 8\}$ & Yes \\
\hline PH-Pfaffian (F) [71] & & $1 / 2$ & $\{0,0,1 / 2,1 / 2,1 / 4,3 / 4\}$ & Yes \\
\hline Anti-Pfaffian (F) [71] & & $-1 / 2$ & $\{0,1 / 2,1 / 4,3 / 4,3 / 8,7 / 8\}$ & Yes \\
\hline
\end{tabular}

open problems in quantum many-body physics continue to defy solution and remain inaccessible for QMC. These include the nature of high-temperature superconductivity and the associated repulsive Hubbard model [20,25-27], dense nuclear matter and the associated lattice QCD at finite baryon density [28-30], and the enigmatic fractional quantum Hall state at filling 5/2 and its associated Coulomb Hamiltonian [31-36], all of which are fermionic.

One may wonder if there is a fundamental reason that no design principle applying to the above open problems has so far been found, despite intense research efforts. More generally,

Are there phases of matter which do not admit a sign-free representative? Are there physical properties that cannot be exhibited by sign-free models?

We refer to such phases of matter, where the sign problem simply cannot be cured, as having an intrinsic sign problem [9]. From a practical perspective, intrinsic sign problems may prove useful in directing research efforts and computational resources. From a fundamental perspective, intrinsic sign problems identify certain phases of matter as inherently quantum - their physical properties cannot be reproduced by a partition function with positive Boltzmann weights.

To the best of our knowledge, the first intrinsic sign problem was discovered by Hastings [9], who proved that no stoquastic, commuting projector, Hamiltonians exist for the "doubled semion" phase [37], which is bosonic and topologically ordered. In a parallel work [38], we generalize this result considerably-excluding the possibility of stoquastic Hamiltonians in a broad class of bosonic nonchiral topological phases of matter. Additionally, Ref. [39] demonstrated, based on the algebraic structure of edge excitations, that no translationally invariant stoquastic Hamiltonians exist for bosonic chiral topological phases.
In this paper, we establish a new criterion for intrinsic sign problems in chiral topological matter, and take the first step in analyzing intrinsic sign problems in fermionic systems. First, based on the well established "momentum polarization" method for characterizing chiral topological matter [40-44], we obtain a variant of the result of Ref. [39]—excluding the possibility of stoquastic Hamiltonians in a broad class of bosonic chiral topological phases. We then develop a formalism with which we obtain analogous results for systems comprising both bosons and fermions-excluding the possibility of sign-free DQMC simulations.

All of the above mentioned topological phases are gapped, 2+1-dimensional, and described at low energy by a topological field theory [45-47]. The class of such phases in which we find an intrinsic sign problem is defined in terms of robust data characterizing them: the chiral central charge $c$, a rational number, as well as the set $\left\{\theta_{a}\right\}$ of topological spins of anyons, a subset of roots of unity. Namely, we find that

$$
\begin{aligned}
& \text { An intrinsic sign problem exists if } e^{2 \pi i c / 24} \text { is not the topological } \\
& \text { spin of some anyon, i.e., } e^{2 \pi i c / 24} \notin\left\{\theta_{a}\right\} \text {. }
\end{aligned}
$$

The above criterion applies to "most" chiral topological phases; see Table I for examples. In particular, we identify an intrinsic sign problem in $96.7 \%$ of the first 1000 fermionic Laughlin phases, in all chiral triplet superconductors, and in the three non-Abelian candidate phases for the quantum Hall state at filling $5 / 2$. We also find intrinsic sign problems in $91.6 \%$ of the first $1000 \mathrm{SU}(2)_{k}$ Chern-Simons theories. Since, for $k \neq 1,2,4$, these allow for universal quantum computation by manipulation of anyons $[48,49]$, our results support the strong belief that quantum computation cannot be simulated with classical resources, in polynomial time [50]. This conclusion is strengthened by examining the Fibonacci anyon model, which is known to be universal for quantum computation [49], and is found to be intrinsically sign-problematic. 
We stress that both $c$ and $\left\{\theta_{a}\right\}$ have clear observable signatures in both the bulk and boundary of chiral topological matter, some of which have been experimentally observed. The chiral central charge controls the boundary thermal Hall conductance [51-54], which was recently measured in quantum Hall and spin systems [31,55-57]. In the bulk it is predicted to contribute to the Hall (or odd) viscosity at finite wave vector, as well as in curved background [58-61]. The chiral central charge also affects the angular momentum at conical defects [62], as was recently observed in an optical realization of integer quantum Hall states $[63,64]$. The topological spins determine the exchange statistics of anyons, predicted to appear in interferometry experiments [49], though experimental observation remains elusive [65]. A measurement of anyonic statistics via current correlations [66] was recently reported in the Laughlin $1 / 3$ quantum Hall state [67].

The paper is organized as follows. In Sec. II we collect relevant facts regarding chiral topological matter, arriving at the "momentum polarization", Eq. (3). Section III then obtains Result 1-an intrinsic sign problem in bosonic chiral topological matter. In Sec. IV we perform a similar analysis for the case where chirality (or time-reversal symmetry breaking), appears spontaneously rather than explicitly, arriving at Result 2. We then turn to fermionic systems. In Sec. V we develop a formalism which unifies and generalizes the currently used DQMC algorithms, and the corresponding design principles. In Sec. VI we obtain within this formalism Result $1 \mathrm{~F}$ and Result 2F, the fermionic analogs of Result 1 and Result 2. Section VII describes a conjectured extension of our results that applies beyond chiral phases and unifies them with the intrinsic sign problems found in our parallel work [38]. In Sec. VIII we discuss our results and provide an outlook for future work.

\section{CHIRAL TOPOLOGICAL MATTER AND SIGNS FROM GEOMETRIC MANIPULATIONS}

In this section we review the necessary details regarding chiral topological phases of matter, and, following Refs. [40-44], obtain the "momentum polarization", Eq. (3), which is the main tool we use in the this paper. Later we will show that gapped bosonic and fermionic Hamiltonians which are sign-free (due to the an appropriate design principle) cannot obey Eq. (3), unless the chiral central charge and topological spins obey a tight constraint.

\section{A. Chiral topological matter}

A gapped Hamiltonian is said to be in a topological phase of matter if it cannot be deformed to a trivial reference Hamiltonian, without closing the gap. If a symmetry is enforced, only symmetric deformations are considered, and it is additionally required that the symmetry is not spontaneously broken [72,73]. For Hamiltonians defined on a lattice, as considered in this paper, a natural trivial Hamiltonian is given by the atomic limit of decoupled lattice sites, where the symmetry acts independently on each site.

Topological phases with a unique ground state on the two-dimensional torus exist only with a prescribed symmetry

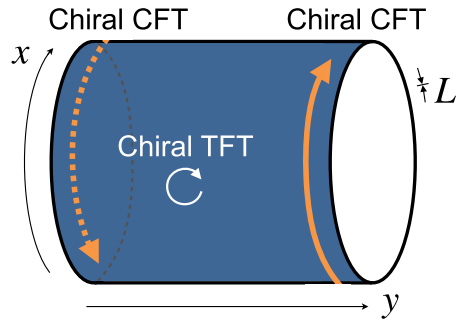

(a) (b)

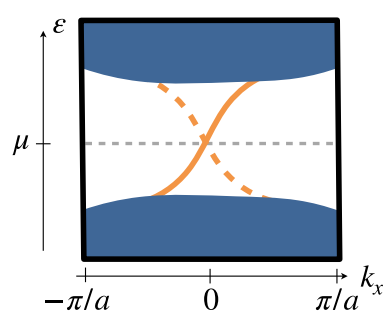

FIG. 1. Chiral topological phases of matter on the cylinder. (a) The low-energy description of a chiral topological phase comprises two, counterpropagating, chiral conformal field theories (CFTs) on the boundary, and a chiral topological field theory (TFT) in the bulk. (b) Examples: schematic single-particle spectrum of a Chern insulator and of the Majorana fermions describing the Kitaev spin liquid. Assuming discrete translational symmetry with spacing $a$ in the $x$ direction, one can plot the single-particle eigenenergies $\varepsilon$ on the cylinder as a function of (quasi) momentum $k_{x}$. This reveals an integer number of chiral dispersion branches whose eigenstates are supported on one of the two boundary components. In the Chern insulator (Kitaev spin liquid) these correspond to the Weyl (Majorana-Weyl) fermion CFT, with $c= \pm 1(c= \pm 1 / 2)$ per branch. The velocity, $v=\left|\partial \varepsilon / \partial k_{x}\right|$ at the chemical potential $\mu$, is a nonuniversal parameter.

group and are termed symmetry-protected topological phases (SPTs) [74-76]. When such phases are placed on the cylinder, they support anomalous boundary degrees of freedom which cannot be realized on isolated one-dimensional spatial manifolds, as well as corresponding quantized bulk response coefficients. Notable examples are the integer quantum Hall states, topological insulators, and topological superconductors [77].

Topological phases with a degenerate ground-state subspace on the torus are termed topologically ordered, or symmetry enriched if a symmetry is enforced $[78,79]$. Beyond the phenomena exhibited by SPTs, these support localized quasiparticle excitations with anyonic statistics and fractional charge under the symmetry group. Notable examples are fractional quantum Hall states [49,80], quantum spin liquids [81], and fractional topological insulators $[82,83]$.

In this work we consider chiral topological phases, where the boundary degrees of freedom that appear on the cylinder propagate unidirectionally. At energies small compared with the bulk gap, the boundary can be described by a chiral conformal field theory (CFT) [84,85], while the bulk reduces to a chiral topological field theory (TFT) [46,47]; see Fig. 1(a). We consider both bosonic and fermionic phases. These may be protected or enriched by an on-site symmetry, but we will not make use of this symmetry in our analysis-only the chirality of the phase will be used.

A notable example for chiral topological phases is given by Chern insulators [86-88]: SPTs protected by the U(1) fermion number symmetry, which admit free-fermion Hamiltonians. The single-particle spectrum of a Chern insulator on the cylinder is depicted in Fig. 1(b). Another notable example is the topologically ordered Kitaev spin liquid [46,89], which can be described by Majorana fermions with a single-particle spectrum similar to Fig. 1(b), coupled to a $\mathbb{Z}_{2}$ gauge field. 
Note that the velocity $v$ of the boundary CFT is a nonuniversal parameter which generically changes as the microscopic Hamiltonian is deformed. Furthermore, different chiral branches may have different velocities.

The chirality of the boundary CFT and bulk TFT is manifested by their nonvanishing chiral central charge $c$, which is rational and universal-it is a topological invariant with respect to continuous deformations of the Hamiltonian which preserve the bulk energy gap, and therefore constant throughout a topological phase $[46,60,90,91]$. On the boundary $c$ is defined with respect to an orientation of the cylinder, so the two boundary components have opposite chiral central charges.

\section{B. Boundary finite-size corrections}

The nonvanishing of $c$ implies a number of geometric, or "gravitational", physical phenomena [58-61,84,85,92,93]. In particular, the boundary supports a nonvanishing energy current $J_{E}$, which receives a correction

$$
J_{E}(T)=J_{E}(0)+2 \pi T^{2} \frac{c}{24},
$$

at a temperature $T>0$, and in the thermodynamic limit $L=$ $\infty$, where $L$ is the circumference of the cylinder. Note that we set $K_{\mathrm{B}}=1$ and $\hbar=1$ throughout. Within CFT, this correction is universal since it is independent of $v$. Taking the two counterpropagating boundary components of the cylinder into account, and placing these at slightly different temperatures, leads to a thermal Hall conductance $K_{H}=c \pi T / 6$ [51-53], a prediction that recently led to the first measurements of $c$ [31,55-57].

In analogy with Eq. (1), the boundary of a chiral topological phase also supports a nonvanishing ground state (at $T=0$ ) momentum density $p$, which receives a universal correction on a cylinder with finite circumference $L<\infty$,

$$
p(L)=p(\infty)+\frac{2 \pi}{L^{2}}\left(h_{0}-\frac{c}{24}\right) .
$$

Equation (2) is the main property of chiral topological matter that we use below, so we discuss it in detail. First, the rational number $h_{0}$ is a chiral conformal weight, which is an additional piece of data characterizing the boundary CFT. Like the chiral central charge, the two boundary components of the cylinder have opposite $h_{0} \mathrm{~s}$. From the bulk TFT perspective, $h_{0}$ corresponds to the topological spin of an anyon quasiparticle, defined by the phase $\theta_{0}=e^{2 \pi i h_{0}}$ accumulated as the anyon undergoes a $2 \pi$ rotation [46]. The set $\left\{\theta_{a}\right\}_{a=1}^{N}$ of topological spins of anyons is associated with the $\mathrm{N}$-dimensional ground-state subspace on the torus, and the unique $\theta_{0}=e^{2 \pi i h_{0}}$ defined by (2) corresponds to the generically unique ground state on the cylinder, with a finite-size energy separation $\sim 1 / L$ from the low-lying excited states; see Appendix A.

As the equation $\theta_{0}=e^{2 \pi i h_{0}}$ suggests, only $h_{0} \bmod 1$ is universal for a topological phase. The integer part of $h_{0}$ can change as the Hamiltonian is deformed on the cylinder, while maintaining the bulk gap, and even as a function of $L$ for a fixed Hamiltonian. Additionally, the choice of $\theta_{0}$ from the set $\left\{\theta_{a}\right\}$ is nonuniversal and can change due to bulk gappreserving deformations or as a function of $L$. Both types (a)
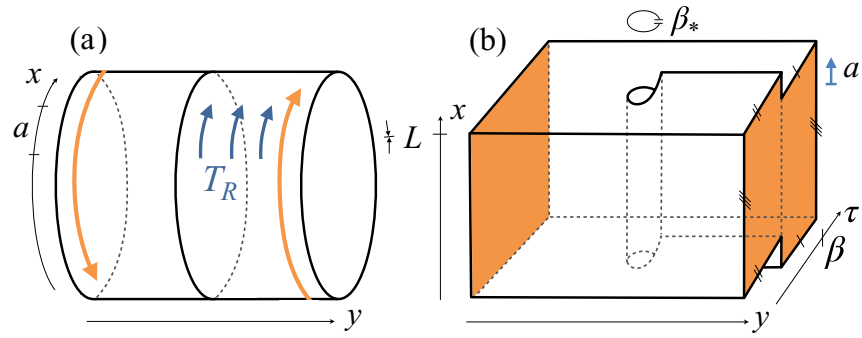

FIG. 2. Momentum polarization. (a) Hamiltonian, or spatial, point of view. The operator $T_{R}$ translates the right half of the cylinder by one unit cell, a distance $a$, in the $x$ direction. It acts as the identity on the left boundary component, and as a translation on the right boundary component. The object $\tilde{Z} / Z$ is the thermal expectation value of $T_{R}$. (b) Field theory, or space-time, point of view. The object $\tilde{Z}$ is the partition function on a space-time carrying a screw dislocation. The space-time region occupied by the boundary components of the spatial cylinder is colored in orange. The screw dislocation can be described as an additional boundary component, on which $T_{R}$ acts as a translation, with a high effective temperature $1 / \beta_{*}$.

of discontinuous jumps in $h_{0}$ may be accompanied by an accidental degeneracy of the ground state on the cylinder. Therefore, the universal and $L$-independent statement regarding $h_{0}$ is that, apart from accidental degeneracies, $e^{2 \pi i h_{0}}=$ $\theta_{0} \in\left\{\theta_{a}\right\}$ - a fact that will be important in our analysis.

The nontrivial behavior of $h_{0}$ described above appears when the boundary corresponds to a nonconformal deformation of a CFT, by, e.g., a chemical potential. As demonstrated analytically and numerically in Appendix B, such behavior appears already in the simple context of Chern insulators with nonzero Fermi momenta, as would be the case in Fig. 1(b) if the chemical potential $\mu$ is either raised or lowered.

\section{Momentum polarization}

In this section we describe a procedure for the extraction of $h_{0}-c / 24$ in Eq. (2), given a lattice Hamiltonian on the cylinder. Since the two boundary components carry opposite momentum densities, the ground state on the cylinder does not carry a total momentum, only a "momentum polarization". It is therefore clear that some sort of one-sided translation will be required.

Following Ref. [40], we define $\tilde{Z}:=\operatorname{Tr}\left(T_{R} e^{-\beta H}\right)$, which is related to the usual partition function $Z=\operatorname{Tr}\left(e^{-\beta H}\right)(\beta=$ $1 / T)$, by the insertion of the operator $T_{R}$, which translates the right half of the cylinder by one unit cell in the periodic $x$ direction; see Fig. 2(a). The object $\tilde{Z}$ satisfies

$$
\tilde{Z}=Z \exp \left[\alpha N_{x}+\frac{2 \pi i}{N_{x}}\left(h_{0}-\frac{c}{24}\right)+o\left(N_{x}^{-1}\right)\right],
$$

where $N_{x}$ is the number of sites in the $x$ direction, $\alpha \in \mathbb{C}$ is nonuniversal and has a negative real part, and $o\left(N_{x}^{-1}\right)$ indicates corrections that decay faster than $N_{x}^{-1}$ as $N_{x} \rightarrow \infty$. Equation (3) is valid at temperatures low compared to the finite-size energy differences on the boundary, $\beta^{-1}=o\left(N_{x}^{-1}\right)$; see Appendix A.

Equation (3) follows analytically from the low-energy description of chiral topological matter in terms of chiral TFT and CFT [40] and was numerically scrutinized in a large 
number of examples in Refs. [40-44], as well as in Appendix B. Nevertheless, we are not aware of a rigorous proof of Eq. (3) for gapped lattice Hamiltonians. Therefore, in stating our results we will use the assumption "the Hamiltonian $H$ is in a chiral topological phase of matter", the content of which is that $H$ admits a low-energy description in terms of a chiral TFT with chiral central charge $c$ and topological spins $\left\{\theta_{a}\right\}$, and in particular, Eq. (3) holds for any bulk-gap preserving deformation of $H$, with $e^{2 \pi i h_{0}} \in\left\{\theta_{a}\right\}$, apart from accidental degeneracies on the cylinder. In the remainder of this section we further discuss the content of Eq. (3) and its expected range of validity, in light of the Hamiltonian and space-time interpretations of $\tilde{Z}$.

From a Hamiltonian perspective, $\tilde{Z} / Z$ is the thermal expectation value of $T_{R}$, evaluated at a temperature $\beta^{-1}$ low enough to isolate the ground state. The exponential decay expressed in Eq. (3) appears because $T_{R}$ is not a symmetry of $H$, and $-\operatorname{Re}(\alpha)$ can be understood as the energy density of the line defect where $T_{R}$ is discontinuous; see Fig. 2(a). In fact, we expect Eq. (3) to hold irrespective of whether the uniform translation is a symmetry of $H$ or of the underlying "lattice" on which $H$ is defined, which may be any polygonalization of the cylinder (see Ref. [94] for a similar scenario). The only expected requirement is that the low energy description of $H$ is homogeneous. Furthermore, if Eq. (3) holds only after a disorder averaging of $\tilde{Z} / Z$, our results and derivations in the following sections remain unchanged.

There is also a simple space-time interpretation of $\tilde{Z}$, which will be useful in the context of DQMC. The usual partition function $Z=\operatorname{Tr}\left(e^{-\beta H}\right)$ has a functional integral representation in terms of bosonic fields $\phi$ (fermionic fields $\psi$ ) defined on space, the cylinder $C$ in our case, and the imaginary time circle $S_{\beta}^{1}=\mathbb{R} / \beta \mathbb{Z}$, with periodic (antiperiodic) boundary conditions [95]. In $\tilde{Z}=\operatorname{Tr}\left(T_{R} e^{-\beta H}\right)$, the insertion of $T_{R}$ produces a twisting of the boundary conditions of $\phi, \psi$ in the time direction, such that $\tilde{Z}$ is the partition function on a space-time carrying a screw dislocation; see Fig. 2(b).

The above interpretation of $\tilde{Z}$, supplemented by Eq. (2), allows for an intuitive explanation of Eq. (3), which loosely follows its analytic derivation [40]. As seen in Fig. 2(b), the line where $T_{R}$ is discontinuous can be interpreted as an additional boundary component at a high effective temperature, $\beta_{*} \ll L / v$. Since the effective temperature is much larger than the finite-size energy differences $2 \pi v / L$ on the boundary CFT, the screw dislocation contributes no finite-size corrections to $\tilde{Z}$. This leaves only the contribution of the boundary component on the right side of the cylinder, where $T_{R}$ produces the phase $e^{i a L p(L)}$, assuming $\beta_{*} \ll L / v \ll \beta$. Equation (2) then leads to the universal finite-size correction $\left(2 \pi i / N_{x}\right)\left(h_{0}-c / 24\right)$.

\section{EXCLUDING STOQUASTIC HAMILTONIANS FOR CHIRAL TOPOLOGICAL MATTER}

In this section we consider bosonic (or "qudit", or spin) systems, and a single design principle - existence of a local basis in which the many-body Hamiltonian is stoquastic. A sketch of the derivation of Result 1 is that the momentum polarization $\tilde{Z}$ is positive for Hamiltonians $H^{\prime}$ which are stoquastic in an on-site and homogenous basis, and this implies that $\theta_{0}=e^{2 \pi i c / 24}$ for any Hamiltonian $H$ obtained from $H^{\prime}$ by conjugation with a local unitary.

\section{A. Setup}

The many-body Hilbert space is given by $\mathcal{H}=\otimes_{\mathbf{x} \in X} \mathcal{H}_{\mathbf{x}}$, where the tensor product runs over the sites $\mathbf{x}=(x, y)$ of a two-dimensional lattice $X$, and $\mathcal{H}_{\mathbf{x}}$ are on-site "qudit" Hilbert spaces of finite dimension $d \in \mathbb{N}$. With finite-size QMC simulations in mind, we consider a square lattice with spacing $1, N_{x} \times N_{y}$ sites, and periodic boundary conditions, so that $X=\mathbb{Z}_{N_{x}} \times \mathbb{Z}_{N_{y}}$ is a discretization of the flat torus $\left(\mathbb{R} / N_{x} \mathbb{Z}\right) \times$ $\left(\mathbb{R} / N_{y} \mathbb{Z}\right)$. Generalization to other two-dimensional lattices is straightforward. On this Hilbert space a gapped $r$-local Hamiltonian $H=\sum_{\mathbf{x}} H_{\mathbf{x}}$, which is in a chiral topological phase of matter, is assumed to be given. Here the terms $H_{\mathbf{x}}$ are supported within a range $r$ of $\mathbf{x}$-they are defined on $\otimes_{|\mathbf{y}-\mathbf{x}| \leqslant r} \mathcal{H}_{\mathbf{y}}$ and act as 0 on all other qudits.

Fix an tensor product basis $|s\rangle=\otimes_{\mathbf{x} \in X}\left|s_{\mathbf{x}}\right\rangle$, labeled by strings $s=\left(s_{\mathbf{x}}\right)_{\mathbf{x} \in X}$, where $s_{\mathbf{x}} \in\{1, \ldots, \mathrm{d}\}$ labels a basis $\left|s_{\mathbf{x}}\right\rangle$ for $\mathcal{H}_{\mathbf{x}}$. For any vector $\mathbf{d} \in X$, the corresponding translation operator $T^{\mathbf{d}}$ is defined in this basis, $T^{\mathbf{d}}|s\rangle=\left|t^{\mathbf{d}} s\right\rangle$, with $\left(t^{\mathbf{d}} s\right)_{\mathbf{x}}=s_{\mathbf{x}+\mathbf{d}}$. These statements assert that $|s\rangle$ is both an onsite and a homogeneous basis, or on-site homogeneous for short. Note that $T^{\mathbf{d}}$ acts as a permutation matrix on the $|s\rangle \mathrm{s}$, and in particular, has non-negative matrix elements in this basis.

In accordance with Sec. IIC, we assume that the low energy description of $H$ is invariant under $T^{\mathbf{d}}$, as defined above. In doing so, we exclude the possibility of generic background gauge fields for any on-site symmetry that $H$ may posses, which is beyond the scope of this work. Nevertheless, commonly used background gauge fields, such as those corresponding to uniform magnetic fields with rational flux per plaquette, can easily be incorporated into our analysis, by restricting to translation vectors $\mathbf{d}$ in a sublattice of $X$. A restriction to sublattice translations can also be used to guarantee that $T^{\mathbf{d}}$ acts purely as a translation in the lowenergy TQFT description. In particular, a lattice translation may permute the anyon types $a .{ }^{1}$ Since the number of anyons is finite, restricting to large enough translations will eliminate this effect. An example is given by Wen's plaquette model, where different anyons are localized on the even or odd sites of a bipartite lattice [96], and a restriction to translations that maps the even (odd) sites to themselves will be made.

Finally, it is assumed that $H$ is locally stoquastic: it is termwise stoquastic in a local basis. This means that a local unitary operator $U$ exists, such that the conjugated Hamiltonian $H^{\prime}=U H U^{\dagger}$ is a sum of local terms $H_{\mathbf{x}}^{\prime}=U H_{\mathbf{x}} U^{\dagger}$, which have nonpositive matrix elements in the on-site homogeneous basis, $\left\langle s\left|H_{\mathbf{x}}^{\prime}\right| \tilde{s}\right\rangle \leqslant 0$ for all basis states $|s\rangle,|\tilde{s}\rangle$. Note that we include the diagonal matrix elements in the definition, without loss of generality.

The term local unitary used above refers to a depth- $D$ quantum circuit, a product $U=U_{D} \cdots U_{1}$ where each $U_{i}$ is itself a

\footnotetext{
${ }^{1}$ We thank Michael Levin for pointing out this phenomenon.
} 


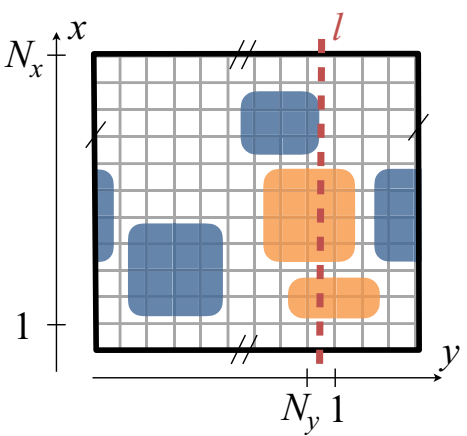

FIG. 3. Cutting the torus to a cylinder along the line $l$. Orange areas mark the supports of Hamiltonian terms $H_{\mathbf{x}}^{\prime}$ which are removed from $H^{\prime}$, while blue areas mark the supports of terms which are kept.

product of unitary operators with nonoverlapping supports ${ }^{2}$ of diameter $w$. It follows that $H^{\prime}$ has a range $r^{\prime}=r+2 r_{U}$, where $r_{U}=D w$ is the range of $U$. Equivalently, we may take $U$ to be a finite-time evolution with respect to an $\tilde{r}$-local, smoothly time-dependent, Hamiltonian $\tilde{H}(t)$, given by the time-ordered exponential $U=\mathrm{TO} e^{-i \int_{0}^{1} \tilde{H}(t) d t}$. The two types of locality requirements are equivalent, as finite-time evolutions can be efficiently approximated by finite-depth circuits, while finitedepth circuits can be written as finite-time evolutions over time $D$ with piecewise constant $w$-local Hamiltonians [73,97].

\section{B. Constraining $c$ and $\left\{\theta_{a}\right\}$}

In order to discuss the momentum polarization, we need to map the stoquastic Hamiltonian $H^{\prime}$ from the torus $X$ to a cylinder $C$. This is done by choosing a translation vector $\mathbf{d} \in$ $X$, and then cutting the torus $X$ along a line $l$ parallel to $\mathbf{d}$. To simplify the presentation we restrict attention to the case $\mathbf{d}=$ $(1,0)$. All other cases amount to a lattice-spacing redefinition; see Appendix C. The cylinder $C=\mathbb{Z}_{N_{x}} \times\left\{1, \ldots, N_{y}\right\}$ is then obtained from the torus $X=\mathbb{Z}_{N_{x}} \times \mathbb{Z}_{N_{y}}$ by cutting along the line $l=\left\{(i, 1 / 2): i \in \mathbb{Z}_{N_{x}}\right\}$. A stoquastic Hamiltonian on the cylinder can be obtained from that on the torus by removing all local terms $H_{\mathbf{x}}^{\prime}$ whose support overlaps $l$; see Fig. 3. Note that this procedure may render $H^{\prime}$ acting as 0 on certain qudits $\mathcal{H}_{\mathbf{x}}$ with $\mathbf{x}$ within a range $r^{\prime}$ of $l$, but this does not bother us. Since all terms $H_{\mathbf{x}}^{\prime}$ are individually stoquastic, this procedure leaves $H^{\prime}$, now defined on the cylinder, stoquastic. One can similarly map $H$ and $U$ to the cylinder $C$ such that the relation $H^{\prime}=U H U^{\dagger}$ remains valid on $C$.

Let us now make contact with the momentum polarization Eq. (3). Having mapped $H^{\prime}$ to the cylinder, we consider the "partition function"

$$
\tilde{Z}^{\prime}:=\operatorname{Tr}\left(e^{-\beta H^{\prime}} T_{R}\right),
$$

where $T_{R}=T_{R}^{\mathbf{d}}$ is defined by $T_{R}|s\rangle=\left|T_{R} s\right\rangle, \quad\left(T_{R} s\right)_{x, y}=$ $s_{x+\Theta(y), y}$, and $\Theta$ is a heavy side function supported on the right half of the cylinder. Though $\tilde{Z}^{\prime}$ is generally different from

\footnotetext{
${ }^{2}$ The support of a unitary $u=e^{i h}$ is the support of its Hermitian generator $h$.
}

$\tilde{Z}=\operatorname{Tr}\left(e^{-\beta H} T_{R}\right)$ appearing in Eq. (3), it satisfies two useful properties:

(1) $\tilde{Z}^{\prime}>0$. Both $-H^{\prime}$ and $T_{R}$ have non-negative entries in the on-site basis $|s\rangle$, and therefore so does $e^{-\beta H^{\prime}} T_{R}$.

(2) $\tilde{Z}^{\prime}$ satisfies Eq. (3), with nonuniversal $\alpha^{\prime}$ in place of $\alpha$, but $c^{\prime}=c$, and $e^{2 \pi i h_{0}^{\prime}} \in\left\{\theta_{a}\right\}$. This follows from the fact that $H^{\prime}=U H U^{\dagger}$ is in the same phase of matter as $H$, and therefore $c^{\prime}=c$, and $\left\{\theta_{a}^{\prime}\right\}=\left\{\theta_{a}\right\}$. Indeed, treating $U$ as a finite-time evolution, we have $H(\lambda)=U(\lambda) H U(\lambda)^{\dagger}$, where $U(\lambda):=\mathrm{TO} e^{-i \int_{0}^{\lambda} \tilde{H}(t) d t}$, as a deformation from $H$ to $H^{\prime}$ which maintains locality and preserves the bulk gap. In fact, since the full spectrum on the cylinder is $\lambda$-independent, we have $h_{0}^{\prime}=h_{0}$ for all $N_{x}$.

Combining the two above properties leads to

$$
1=\tilde{Z}^{\prime} /\left|\tilde{Z}^{\prime}\right|=\exp 2 \pi i\left[\epsilon^{\prime} N_{x}+\frac{1}{N_{x}}\left(h_{0}-\frac{c}{24}\right)+o\left(N_{x}^{-1}\right)\right],
$$

where $\epsilon^{\prime}:=\operatorname{Im}\left(\alpha^{\prime}\right) / 2 \pi$. The nonuniversal integer part of $h_{0}$ can then be eliminated by raising Eq. (5) to the $N_{x}$ th power,

$$
1=e^{2 \pi i \epsilon^{\prime} N_{x}^{2}} \theta_{0}\left(N_{x}\right) e^{-2 \pi i c / 24}+o(1)
$$

where we used $\theta_{0}=e^{2 \pi i h_{0}}$, and $o(1) \rightarrow 0$ as $N_{x} \rightarrow \infty$. We also indicated explicitly the possible $N_{x}$ dependence of $\theta_{0}$, as described in Sec. II B. We proceed under the assumption that no accidental degeneracies occur on the cylinder, so that $\theta_{0}\left(N_{x}\right) \in\left\{\theta_{a}\right\}$ for all $N_{x}$, deferring the degenerate case to Appendix D. Now, for rational $\epsilon^{\prime}=n / m$, the series $e^{2 \pi i \epsilon^{\prime} N_{x}^{2}}$ $\left(N_{x} \in \mathbb{N}\right)$ covers periodically a subset $S$ of the $m$ th roots of unity, including $1 \in S$. On the other hand, for irrational $\epsilon^{\prime}$ the series $e^{2 \pi i \epsilon^{\prime} N_{x}^{2}}$ is dense in the unit circle. Combined with the fact that $\theta_{0}\left(N_{x}\right)$ is valued in the finite set $\left\{\theta_{a}\right\}$, while $c$ is $N_{x}$-independent, Eq. (6) implies that $\epsilon^{\prime}$ must be rational, and that the values attained by $\theta_{0}\left(N_{x}\right) e^{-2 \pi i c / 24}$ cover the set $S$ periodically, for large enough $N_{x}$. It follows that $1 \in S \subset$ $\left\{\theta_{a} e^{-2 \pi i c / 24}\right\}$. We therefore have the following result:

Result 1. If a local bosonic Hamiltonian $H$ is both locally stoquastic and in a chiral topological phase of matter, then one of the corresponding topological spins satisfies $\theta_{a}=e^{2 \pi i c / 24}$. Equivalently, a bosonic chiral topological phase of matter where $e^{2 \pi i c / 24}$ is not the topological spin of some anyon, i.e., $e^{2 \pi i c / 24} \notin\left\{\theta_{a}\right\}$, admits no local Hamiltonians which are locally stoquastic.

The above result can be stated in terms of the topological T matrix, which is the representation of a Dehn twist on the torus ground-state subspace and has the spectrum $\operatorname{Spec}(\mathbf{T})=$ $\left\{\theta_{a} e^{-2 \pi i c / 24}\right\}_{a}[40,41,45,46,80,98]$ :

Result $1^{\prime}$. If a local bosonic Hamiltonian $H$ is both locally stoquastic and in a chiral topological phase of matter, then the corresponding $\mathbf{T}$ matrix satisfies $1 \in \operatorname{Spec}(\mathbf{T})$. Equivalently, a bosonic chiral topological phase of matter where $1 \notin \operatorname{Spec}(\mathbf{T})$, admits no local Hamiltonians which are locally stoquastic.

The above result is our main statement for bosonic phases of matter. The logic used in its derivation will be extended 
in the following sections, where we generalize Result 1 to systems which are fermionic, spontaneously chiral, or both.

\section{SPONTANEOUS CHIRALITY}

The invariants $h_{0}$ and $c$ change sign under both time reversal $\mathcal{T}$ and parity (spatial reflection) $\mathcal{P}$, and therefore require a breaking of $\mathcal{T}$ and $\mathcal{P}$ down to $\mathcal{P} \mathcal{T}$ to be nonvanishing. The momentum polarization Eq. (3) is valid if this symmetry breaking is explicit, i.e., $H$ does not commute with $\mathcal{P}$ and $\mathcal{T}$ separately. Here we consider the case where $H$ is $\mathcal{P}, \mathcal{T}$ symmetric, but these are broken down to $\mathcal{P} \mathcal{T}$ spontaneously, as in, e.g., intrinsic topological superfluids and superconductors $[61,99,100]$. We first generalize Eq. (3) to this setting, and then use this generalization to obtain a spontaneously chiral analog of Result 1 .

Note that the physical time-reversal $\mathcal{T}$ is an on-site antiunitary operator acting identically on all qudits, which implies $\left[\mathcal{T}, T_{R}\right]=0$, while $\mathcal{P}$ is a unitary operator that maps the qudit at $\mathbf{x}$ to that at $P \mathbf{x}$, where $P$ is the nontrivial element in $O(2) / S O(2)$, e.g., $(x, y) \mapsto(-x, y)$.

\section{A. Momentum polarization for spontaneously chiral Hamiltonians}

For simplicity, we begin by assuming that $H$ is "classically symmetry breaking"-it has two exact ground states on the cylinder, already at finite system sizes. We therefore have two ground states $| \pm\rangle$, such that $|-\rangle$ is obtained from $|+\rangle$ by acting with either $\mathcal{T}$ or $\mathcal{P}$. In particular, $| \pm\rangle$ have opposite values of $h_{0}$ and $c$. The $\beta \rightarrow \infty$ density matrix is then $e^{-\beta H} / Z=\left(\rho_{+}+\rho_{-}\right) / 2$, where $\rho_{ \pm}=| \pm\rangle\langle \pm|$, and this modifies the right-hand side of Eq. (3) to its real part,

$$
\begin{aligned}
\tilde{Z} & :=\operatorname{Tr}\left(T_{R} e^{-\beta H}\right) \\
& =Z e^{-\delta N_{x}} \cos 2 \pi\left[\epsilon N_{x}+\frac{2 \pi}{N_{x}}\left(h_{0}-\frac{c}{24}\right)+o\left(N_{x}^{-1}\right)\right],
\end{aligned}
$$

where $-\delta \pm 2 \pi i \epsilon$ are the values of the nonuniversal $\alpha$ obtained from Eq. (3), by replacing $e^{-\beta H}$ by $\rho_{ \pm}$. Indeed, it follows from $\left[\mathcal{T}, T_{R}\right]=0$ that if two density matrices are related by $\rho_{-}=\mathcal{T} \rho_{+} \mathcal{T}^{-1}$, then $\tilde{Z}_{ \pm}:=Z_{ \pm} \operatorname{Tr}\left(T_{R} \rho_{+}\right)$are complex conjugates, $\tilde{Z}_{-}=\tilde{Z}_{+}^{*}$.

Now, for a generic symmetry-breaking Hamiltonian $H$, exact ground-state degeneracy happens only in the infinite volume limit [101]. At finite size, the two lowest lying eigenvalues of $H$ would be separated by an exponentially small energy difference $\Delta E=O\left(e^{-f N_{x}^{\lambda}}\right)$, with some $f>0, \lambda>0$. The two corresponding eigenstates would be $\mathcal{T}, \mathcal{P}$-even/odd, of the form $W[|+\rangle \pm|-\rangle]$, where $W$ is a $\mathcal{T}, \mathcal{P}$-invariant local unitary [73]. One can think of these statements as resulting from the existence of a bulk-gap preserving and $\mathcal{T}, \mathcal{P}$-symmetric deformation of $H$ to a "classically symmetry-breaking" Hamiltonian. ${ }^{3}$

\footnotetext{
${ }^{3}$ The canonical example is the transverse field Ising model $H(g)=$ $-\sum_{i=1}^{N_{x}}\left(Z_{i} Z_{i+1}+g X_{i}\right)$ in $1+1$ d. Exact ground-state degeneracy appears at finite $N_{x}$ only for $g=0$, though spontaneous symmetry breaking occurs for all $|g|<1$, where a splitting $\sim|g|^{N_{x}}$ appears.
}

In the generic setting, we have

$$
e^{-\beta H} / Z=W\left(\rho_{+}+\rho_{-}\right) W^{\dagger} / 2+O(\beta \Delta E),
$$

and, following our treatment of the local unitary $U$ in the previous section, Equation (7) remains valid, with modified $\delta, \epsilon$, but unchanged $h_{0}-c / 24$. This statement holds for temperatures much higher than $\Delta E$ and much smaller that the CFT energy spacing, $\Delta E \ll \beta^{-1} \ll N_{x}^{-1}$, or more accurately $\beta^{-1}=o\left(N_{x}^{-1}\right)$ and $\beta \Delta E=o\left(N_{x}^{-1}\right)$ (cf. Sec. II C). Note that the universal content of Eq. (7) is the absolute value $\left|h_{0}-c / 24\right|$, since the cosine is even and $\operatorname{sgn}(\epsilon)$ is nonuniversal.

\section{B. Constraining $c$ and $\left\{\theta_{a}\right\}$}

Let us now assume that a gapped and local Hamiltonian $H$ is $\mathcal{T}, \mathcal{P}$-symmetric, and is locally stoquastic, due to a unitary $U$. It follows that $\tilde{Z}^{\prime}=\operatorname{Tr}\left(T_{R} e^{-\beta H^{\prime}}\right)>0$, where $H^{\prime}=U H U^{\dagger}$. If $U$ happens to be $\mathcal{T}, \mathcal{P}$-symmetric, then so is $H^{\prime}$, and Eq. (7) holds for $\tilde{Z}^{\prime}$, with $\delta^{\prime}, \epsilon^{\prime}$ in place of $\delta, \epsilon$. For a general $U$, we have

$$
e^{-\beta H^{\prime}} / Z^{\prime}=U W\left(\rho_{+}+\rho_{-}\right) W^{\dagger} U^{\dagger} / 2+O(\beta \Delta E),
$$

where $U W$ need not be $\mathcal{T}, \mathcal{P}$-symmetric. As result, $\tilde{Z}^{\prime}$ satisfies a weaker form of Eq. (7),

$$
0<\tilde{Z}^{\prime}=\left(Z^{\prime} / 2\right) \sum_{\sigma= \pm} e^{-\delta_{\sigma}^{\prime} N_{x}} e^{2 \pi i \sigma\left[\epsilon_{\sigma}^{\prime} N_{x}+\frac{1}{N_{x}}\left(h_{0}-\frac{c}{24}\right)+o\left(N_{x}^{-1}\right)\right]}
$$

where $\delta_{+}^{\prime}, \epsilon_{+}^{\prime}$ may differ from $\delta_{-}^{\prime}, \epsilon_{-}^{\prime}$, and we also indicated the positivity of $\tilde{Z}^{\prime}$. Now, if $\delta_{+}^{\prime} \neq \delta_{-}^{\prime}$, one of the chiral contributions is exponentially suppressed relative to the other as $N_{x} \rightarrow \infty$, and we can apply the analysis of Sec. III. If $\delta_{+}^{\prime}=\delta_{-}^{\prime}$, we obtain

$$
0<\sum_{\sigma= \pm} \exp 2 \pi i \sigma\left[\epsilon_{\sigma}^{\prime} N_{x}+\frac{1}{N_{x}}\left(h_{0}-\frac{c}{24}\right)+o\left(N_{x}^{-1}\right)\right]
$$

in analogy with Eq. (5). Unlike Eq. (5), taking the $N_{x}$ th power of this equation does not eliminate the mod 1 ambiguity in $h_{0}$. This corresponds to the fact that, as opposed to explicitly chiral systems, stacking copies of a spontaneously chiral system does not increase its net chirality. One can replace $T_{R}$ in $\tilde{Z}^{\prime}$ with a larger half-translation $T_{R}^{m}$, which would multiply the argument of the cosine by $m$. However, since the largest translation on the cylinder is obtained for $m \approx N_{x} / 2$, this does not eliminate the mod 1 ambiguity in $h_{0}$. Moreover, even if it so happens that $\epsilon_{+}^{\prime}=\epsilon_{-}^{\prime}=0$, Eq. (11) does not imply $h_{0}-c / 24=0(\bmod 1)$ since $N_{x}$ is large.

In order to make progress, we make use of the bagpipes construction illustrated in Fig. 4. We attach $M$ identical cylinders, or "pipes", to the given lattice, and act with $T_{R}$ on these cylinders. The global topology of the given lattice is unimportant-all that is needed is a large enough disk in which the construction can be applied. The construction does require some form of homogeneity in order to have a unique extension of the Hamiltonian $H^{\prime}$ to the pipes and which will be identical for all pipes. We will assume a strict translation symmetry with respect to a sublattice, but we believe that this assumption can be relaxed. 


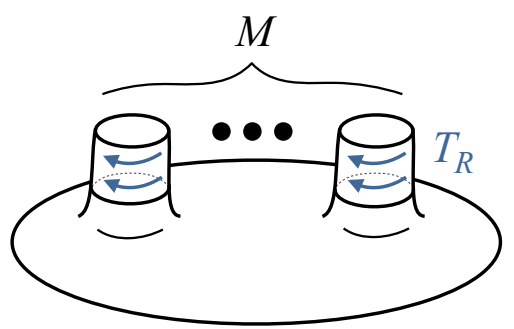

FIG. 4. Bagpipes construction. We attach $M$ identical cylinders, or "pipes", to the given lattice, and define the half translation $T_{R}$ to act on their top halves, as indicated by blue arrows. The contributions of the pipes to the momentum polarization adds, producing the factor $M$ in Eq. (12).

The resulting surface, shown in Fig. 4, has negative curvature at the base of each pipe, which requires a finite number of lattice disclinations in this region. In order to avoid any possible ambiguity in the definition of $H^{\prime}$ at a disclination, one can simply remove any local term $H_{\mathbf{x}}^{\prime}$ whose support contains a disclination, which amounts to puncturing a hole around each disclination. The resulting boundary components do not contribute to the momentum polarization since $T_{R}$ acts on these as the identity.

With the construction at hand, the identical contributions of all cylinders to $\tilde{Z}^{\prime}$ add, which implies

$0<\sum_{\sigma= \pm} \exp 2 \pi i \sigma M\left[\epsilon_{\sigma}^{\prime} N_{x}+\frac{1}{N_{x}}\left(h_{0}-\frac{c}{24}\right)+o\left(N_{x}^{-1}\right)\right]$.

Setting $M=N_{x}$ gives

$0<e^{2 \pi i \epsilon_{+}^{\prime} N_{x}^{2}} \theta_{0}\left(N_{x}\right) e^{-2 \pi i c / 24}+e^{-2 \pi i \epsilon_{-}^{\prime} N_{x}^{2}} \theta_{0}^{*}\left(N_{x}\right) e^{2 \pi i c / 24}+o(1)$,

where we indicate explicitly the possible $N_{x}$ dependence of $\theta_{0}$. This is the spontaneously chiral analog of Eq. (6) and can be analyzed similarly. Since $\theta_{0}\left(N_{x}\right)$ is valued in the finite set $\left\{\theta_{a}\right\}$, both $\epsilon_{ \pm}^{\prime}$ must be rational, $\epsilon_{ \pm}^{\prime}=n_{ \pm} / m_{ \pm}$. Restricting then to $N_{x}=n_{x} m_{+} m_{-}$, such that $e^{2 \pi i \epsilon_{ \pm} N_{x}^{2}}=1$, and $\theta_{0}$ attains a constant value $\theta_{a}$ for large enough $n_{x}$, we have

$$
0<\operatorname{Re}\left(\theta_{a} e^{-2 \pi i c / 24}\right),
$$

for some anyon $a$. Repeating the analysis with $k$ times more pipes $M=k N_{x}$ replaces $\theta_{a} e^{-2 \pi i c / 24}$ in Eq. (14) with its $k$ th power, for all $k \in \mathbb{N}$. This infinite set of equations then implies $\theta_{a} e^{-2 \pi i c / 24}=1$. We summarize with the following result:

Result 2. If a local bosonic Hamiltonian $H$ is both locally stoquastic and in a spontaneously chiral topological phase of matter, then one of the corresponding topological spins satisfies $\theta_{a}=e^{2 \pi i c / 24}$. Equivalently, a bosonic spontaneously chiral topological phase of matter where $e^{2 \pi i c / 24}$ is not the topological spin of some anyon, i.e., $e^{2 \pi i c / 24} \notin\left\{\theta_{a}\right\}$, admits no local Hamiltonians which are locally stoquastic.

This extends Result 1 beyond explicitly chiral Hamiltonians, and clarifies that the essence of the intrinsic sign problem we find is the macroscopic, physically observable, condition $e^{2 \pi i c / 24} \notin\left\{\theta_{a}\right\}$, as opposed to the microscopic absence (or presence) of time-reversal and reflection symmetries.

\section{DQMC: LOCALITY, HOMOGENEITY, AND GEOMETRIC MANIPULATIONS}

In order to obtain fermionic analogs of the bosonic results of the previous sections, we first need to establish a framework in which such results can be obtained. In this section we develop a formalism that unifies and generalizes the currently used DQMC algorithms and design principles, and implement within it the geometric manipulations used in previous sections, in a sign-free manner. Since we wish to treat the wide range of currently known DQMC algorithms and design principles on equal footing, the discussion will be more abstract than the simple setting of locally stoquastic Hamiltonians used above. In particular, Secs. V A-V B lead up to the definition of locally sign-free $D Q M C$, which is our fermionic analog of a locally stoquastic Hamiltonian. This definition is used later on in Sec. VI to formulate Result $1 F$ and Result 2F, the fermionic analogs of Result 1 and 2. The new tools needed to establish these results are the sign-free geometric manipulations described in Sec. V D.

\section{A. Local DQMC}

In the presence of bosons and fermions, the many-body Hilbert space is given by $\mathcal{H}=\mathcal{H}_{\mathrm{F}} \otimes \mathcal{H}_{\mathrm{B}}$, where $\mathcal{H}_{\mathrm{F}}$ is a fermionic Fock space, equipped with an on-site occupation basis $|v\rangle_{\mathrm{F}}=\prod_{\mathbf{x}, \alpha}\left(f_{\mathbf{x}, \alpha}^{\dagger}\right)^{v_{\mathbf{x}, \alpha}}|0\rangle_{\mathrm{F}}, v_{\mathbf{x}, \alpha} \in\{0,1\}$, generated by acting with fermionic (anticommuting) creation operators $f_{\mathbf{x}, \alpha}^{\dagger}$ on the Fock vacuum $|0\rangle_{\mathrm{F}}$. The product is taken with respect to a fixed ordering of fermion species $\alpha \in$ $\left\{1, \ldots, \mathrm{d}_{\mathrm{F}}\right\}$ and lattice sites $\mathbf{x} \in X$. We will also make use of the single-fermion space $\mathcal{H}_{1 \mathrm{~F}} \cong \mathbb{C}^{|X|} \otimes \mathbb{C}^{\mathrm{d}_{\mathrm{F}}}$, spanned by $|\mathbf{x}, \alpha\rangle_{\mathrm{F}}=f_{\mathbf{x}, \alpha}^{\dagger}|0\rangle_{\mathrm{F}}$, where $|X|=N_{x} N_{y}$ is the system size. As in Sec. III, $\mathcal{H}_{\mathrm{B}}$ is a many-qudit Hilbert space with local dimension $d$. It can also be a bosonic Fock space where $d=\infty$.

We consider local fermion-boson Hamiltonians $H$, of the form

$$
H=\sum_{\mathbf{x}, \mathbf{y}} f_{\mathbf{x}}^{\dagger} h_{0}^{\mathbf{x}, \mathbf{y}} f_{\mathbf{y}}+H_{I},
$$

where the free-fermion Hermitian matrix $h_{0}^{\mathbf{x}, \mathbf{y}}$ is $r_{0}$-local, it vanishes unless $|\mathbf{x}-\mathbf{y}| \leqslant r_{0}$, and we suppress, here and in the following, the fermion species indices. The Hamiltonian $H_{I}$ describes all possible $r_{0}$-local interactions which preserve the fermion parity $(-1)^{N_{f}}$, where $N_{f}=\sum_{\mathbf{x}} f_{\mathbf{x}}^{\dagger} f_{\mathbf{x}}$, including fermion-independent terms $H_{\mathrm{B}}$ as in Sec. III. Thus $H_{I}$ is of the form

$$
H_{I}=H_{\mathrm{B}}+\sum_{\mathbf{x}, \mathbf{y}} f_{\mathbf{x}}^{\dagger} K_{\mathrm{B}}^{\mathbf{x}, \mathbf{y}} f_{\mathbf{y}}+\sum_{\mathbf{x}, \mathbf{y}, \mathbf{z}, \mathbf{w}} f_{\mathbf{x}}^{\dagger} f_{\mathbf{y}}^{\dagger} V_{\mathrm{B}}^{\mathbf{x}, \mathbf{y}, \mathbf{z}, \mathbf{w}} f_{\mathbf{z}} f_{\mathbf{w}}+\cdots,
$$

where $K_{\mathrm{B}}^{\mathbf{x}, \mathbf{y}}$ (for all $\mathbf{x}, \mathbf{y} \in X$ ) is a local bosonic operator with range $r_{0}$, and vanishes unless $|\mathbf{x}-\mathbf{y}| \leqslant r_{0}$, and similarly for $V_{\mathrm{B}}^{\mathbf{x}, \mathbf{y}, \mathbf{z}, \mathbf{w}}$, which vanishes unless $\mathbf{x}, \mathbf{y}, \mathbf{z}, \mathbf{w}$ are contained in a disk or radius $r_{0}$. In Eq. (15) dots represent additional pairing terms of the form $f f, f^{\dagger} f^{\dagger}$, or $f f f f, f^{\dagger} f^{\dagger} f^{\dagger} f^{\dagger}$, as well as terms with a higher number of fermions, all of which are $r_{0}$ local and preserve the fermion parity.

Since locality is defined in terms of anticommuting Fermi operators, a local stoquastic basis is not expected to exist, and 
accordingly, the sign problem appears in any QMC method in which the Boltzmann weights are given in terms of Hamiltonian matrix elements in a local basis [2,5]. For this reason, the methods used to perform QMC in the presence of fermions are distinct from the ones used in their absence. These are collectively referred to as DQMC $[4,5,17,19,20]$ and lead to the imaginary time path integral representation of the partition function $Z=\operatorname{Tr}\left(e^{-\beta H}\right)$,

$$
\begin{aligned}
Z & =\int D \phi D \psi e^{-S_{\phi}-S_{\psi, \phi}} \\
& =\int D \phi e^{-S_{\phi}} \operatorname{Det}\left(D_{\phi}\right) \\
& =\int D \phi e^{-S_{\phi}} \operatorname{Det}\left(I+U_{\phi}\right),
\end{aligned}
$$

involving a bosonic field $\phi$ with an action $S_{\phi}$, and a fermionic (Grassmann-valued) field $\psi$, with a quadratic action $S_{\psi, \phi}=\sum_{\mathbf{x}, \mathbf{x}^{\prime}} \int \mathrm{d} \tau \bar{\psi}_{\mathbf{x}, \tau}\left[D_{\phi}\right]_{\mathbf{x}, \mathbf{y}} \psi_{\mathbf{y}, \tau}$ defined by the $\phi$ dependent single-fermion operator $D_{\phi}$. In the third line of Eq. (17) we assumed the Hamiltonian form $D_{\phi}=\partial_{\tau}+h_{\phi(\tau)}$, and used a standard identity for the determinant in terms of the single-fermion imaginary-time evolution operator $U_{\phi}=$ $\mathrm{TO} e^{-\int_{0}^{\beta} h_{\phi(\tau)} \mathrm{d} \tau}[19]$, where TO denotes the time ordering. The field $\phi(\psi)$ is defined on a continuous imaginary-time circle $\tau \in \mathbb{R} / \beta \mathbb{Z}$, with periodic (antiperiodic) boundary conditions, and on the spatial lattice $X$. The second and third lines of Eq. (17) define the Monte Carlo phase space $\{\phi\}$ and Boltzmann weight

$$
p(\phi)=e^{-S_{\phi}} \operatorname{Det}\left(D_{\phi}\right)=e^{-S_{\phi}} \operatorname{Det}\left(I+U_{\phi}\right) .
$$

In applications, the DQMC representation (17) may be obtained from the Hamiltonian $H$ in a number of ways. If a Yukawa-type model is assumed as a starting point [17], i.e., $H_{I}=H_{\mathrm{B}}+\sum_{\mathbf{x}, \mathbf{y}} f_{\mathbf{x}}^{\dagger} K_{\mathrm{B}}^{\mathbf{x}, \mathbf{y}} f_{\mathbf{y}}$, then the action $S_{\phi}$ is obtained from the Hamiltonian $H_{\mathrm{B}}$, and $h_{\phi(\tau)}=h_{0}+K_{\mathrm{B}}$. Alternatively, the representation (17) may be obtained through a Hubbard-Stratonovich decoupling and/or a series expansion of fermionic self-interactions $[18,21,102]$. Such is the case, e.g., when there are no bosons $\mathcal{H}=\mathcal{H}_{\mathrm{F}}$, and $H_{I}=$ $\sum f_{\mathbf{x}}^{\dagger} f_{\mathbf{y}}^{\dagger} V^{\mathbf{x}, \mathbf{y}, \mathbf{z}, \mathbf{w}} f_{\mathbf{z}} f_{\mathbf{w}}$. Note that for a given fermionic selfinteraction, there are various possible DQMC representations, obtained, e.g., via a Hubbard-Stratonovich decoupling in different channels.

To take into account and generalize the above relations between $H$ and the corresponding DQMC representation, we will assume only (i) that the effective single-fermion Hamiltonian $h_{\phi(\tau)}$ reduces to the free fermion matrix $h^{(0)}$ in the absence of $\phi$, i.e., $h_{\phi(\tau)=0}=h_{0}$, (ii) that the boson field $\phi$ is itself an $r_{0}$-local object, ${ }^{4}$ and (iii) that the $r_{0}$ locality of $h_{0}$ and $H_{I}$ implies the $r$ locality of $S_{\phi}$ and $h_{\phi(\tau)}$, where $r$ is some function of $r_{0}$, independent of system size. The physical

\footnotetext{
${ }^{4}$ Thus $\phi$ is a map from sets of lattice sites with diameter less than $r_{0}$, such as links, plaquettes, etc., to a fixed vector space $\mathbb{C}^{k}$. Additionally, the $\phi$ integration in (17) runs over all such functions. As an example, restricting to constant functions $\phi$ leads to nonlocal all to all interactions between fermions.
}

content of these assumptions is that the fields $\psi$ and operators $f$ correspond to the same physical fermion, ${ }^{5}$ and that the boson $\phi$ mediates all fermionic interactions $H_{I}$ and therefore corresponds to both the physical bosons in $\mathcal{H}_{\mathrm{B}}$ and to composite objects made of an even number of fermions within a range $r_{0}$ (e.g., a Cooper pair $\phi \sim f f$ ).

We can therefore write

$$
S_{\phi}=\sum_{\tau, \mathbf{x}} S_{\phi ; \tau, \mathbf{x}}, \quad h_{\phi(\tau)}=\sum_{\mathbf{x}} h_{\phi(\tau) ; \mathbf{x}},
$$

where each term $S_{\phi ; \tau, x}$ depends only on the values of $\phi$ at points $\left(\mathbf{x}^{\prime}, \tau^{\prime}\right)$ with $\left|\tau-\tau^{\prime}\right|,\left|\mathbf{x}-\mathbf{x}^{\prime}\right| \leqslant r$, and similarly, each term $h_{\phi(\tau) ; \mathbf{x}}$ is supported on a disk of radius $r$ around $\mathbf{x}$, and depends on the values of $\phi(\tau)$ at points $\mathbf{x}$ within this disk.

Note that even though $H$ is Hermitian, we do not assume the same for $h_{\phi(\tau)}$. Non-Hermitian $h_{\phi(\tau)}$ s naturally arise in Hubbard-Stratonovich decouplings; see, e.g., Refs. [18,103]. Even when $h_{\phi(\tau)}$ is Hermitian for all $\phi$, its time dependence implies that $U_{\phi}$ is non-Hermitian, and therefore $\operatorname{Det}\left(I+U_{\phi}\right)$ in Eq. (18) is generically complex valued [19]. This is the generic origin of the sign problem in DQMC. Section VB describes the notion of fermionic design principles, algebraic conditions on $U_{\phi}$ implying $\operatorname{Det}\left(I+U_{\phi}\right) \geqslant 0$ and defines what it means for such design principles to be local and homogenous.

In the following analysis, we exclude the case of "classically interacting fermions", where $\phi$ is time-independent and $h_{\phi}$ is Hermitian. In this case the fermionic weight $\operatorname{Det}\left(I+e^{-\beta h_{\phi}}\right)$ is trivially non-negative, and sign-free DQMC is always possible, provided $S_{\phi} \in \mathbb{R}$. We view such models as "exactly solvable", on equal footing with free-fermion and commuting projector models. Given a phase of matter, the possible existence of exactly solvable models is independent of the possible existence of sign-free models. Even when an exactly solvable model exists, QMC simulations are of interest for generic questions, such as phase transitions due to deformations of the model [104]. In particular, Ref. [105] utilized a classically free description of Kitaev's honeycomb model to obtain the thermal Hall conductance and chiral central charge, which should be contrasted with the intrinsic sign problem we find in the corresponding phase of matter; see Table I and Sec. VI.

\section{B. Local and homogenous fermionic design principles}

The representation (17) is sign-free if $p(\phi)=$ $e^{-S_{\phi}} \operatorname{Det}\left(I+U_{\phi}\right) \geqslant 0$ for all $\phi$. A design principle then amounts to a set of polynomially verifiable properties ${ }^{6}$ of $S_{\phi}$ and $h_{\phi(\tau)}$ that guarantee that the complex phase of

\footnotetext{
${ }^{5}$ Technically, via the fermionic coherent state construction of the functional integral [95].

${ }^{6}$ That is, properties which can be verified in a polynomial-in$\beta|X|$ time. As an example, given a local Hamiltonian, deciding whether there exists a local basis in which it is stoquastic is NPcomplete [6,7]. In particular, one does not need to perform the exponential operation of evaluating $p$ on every configuration $\phi$ to ensure that $p(\phi) \geqslant 0$. Had this been possible, there would be no need for a Monte Carlo sampling of the phase space $\{\phi\}$.
} 
$\operatorname{Det}\left(I+U_{\phi}\right)$ is opposite to that of $e^{-S_{\phi}}$. For the sake of presentation, we restrict attention to the case where $S_{\phi}$ is manifestly real valued, and $\operatorname{Det}\left(I+U_{\phi}\right) \geqslant 0$ due to an algebraic condition on the operator $U_{\phi}$, which we write as $U_{\phi} \in \mathcal{C}_{U}$. This is assumed to follow from an algebraic condition on $h_{\phi(\tau)}$, written as $h_{\phi(\tau)} \in \mathcal{C}_{h}$, manifestly satisfied for all $\phi(\tau)$. The set $\mathcal{C}_{h}$ is assumed to be closed under addition, while $\mathcal{C}_{U}$ is closed under multiplication: $h_{1}+h_{2} \in \mathcal{C}_{h}$ for all $h_{1}, h_{2} \in \mathcal{C}_{h}$, and $U_{1} U_{2} \in \mathcal{C}_{U}$ for all $U_{1}, U_{2} \in \mathcal{C}_{U}$.

The simplest example, where $\mathcal{C}_{U}=\mathcal{C}_{h}$ is the set of matrices obeying a fixed time-reversal symmetry, is discussed in Sec. V C. In Appendix F we review all other design principles known to us, demonstrate that most of them are of the simplified form above, and generalize our arguments to those that are not. Comparing with the bosonic Hamiltonians treated in Sec. III, we note that $\mathcal{C}_{h}$ is analogous to the set of stoquastic Hamiltonians $H$ in a fixed basis, while $\mathcal{C}_{U}$ is analogous to the resulting set of matrices $e^{-\beta H}$ with non-negative entries.

Design principles, as defined above (and in the literature), are purely algebraic conditions, which carry no information about the underlying geometry of space-time. However, as demonstrated in Sec. V C, in order to allow for local interactions, mediated by an $r_{0}$-local boson $\phi$, a design principle must also be local in some sense. We will adopt the following definitions, which are shown to be satisfied by all physical applications of design principles that we are aware of, in Sec. V C and Appendix F.

Definition (termwise sign-free). We say that a DQMC representation is termwise sign-free due to a design principle $\mathcal{C}_{h}$, if each of the local terms $S_{\phi ; \tau, \mathbf{x}}, h_{\phi(\tau) ; \mathbf{x}}$ obey the design principle separately, rather than just they sums $S_{\phi}, h_{\phi(\tau)}$. Thus $S_{\phi ; \tau, \mathbf{x}}$ is real valued, and $h_{\phi(\tau) ; \mathbf{x}} \in \mathcal{C}_{h}$, for all $\tau, \mathbf{x}$.

This is analogous to the requirement in Sec. III A that $H^{\prime}$ be termwise stoquastic. Note that even when a DQMC representation is termwise sign-free, the resulting Boltzmann weights $p(\phi)$ are sign-free in a nonlocal manner: $\operatorname{Det}\left(I+U_{\phi}\right)$ involves the values of $\phi$ at all space-time points, and splitting the determinant into a product of local terms by the Leibniz formula reintroduces signs, which capture the fermionic statistics. In this respect, the "classical" Boltzmann weights $p(\phi)$ are always nonlocal in DQMC.

Definition (on-site homogeneous design principle). A design principle is said to be on-site homogenous if any permutation of the lattice sites $\sigma \in S_{X}$ obeys it. That is, the operator

$$
O_{(\mathbf{x}, \alpha),\left(\mathbf{x}^{\prime}, \alpha^{\prime}\right)}^{(\sigma)}=\delta_{\mathbf{x}, \sigma\left(\mathbf{x}^{\prime}\right)} \delta_{\alpha, \alpha^{\prime}},
$$

viewed as a single-fermion imaginary-time evolution operator, obeys the design principle: $O^{(\sigma)} \in \mathcal{C}_{U}$, for all $\sigma \in S_{X}$.

This amounts to the statement that the design principle treats all lattice sites on equal footing, since it follows that $U_{\phi} \in \mathcal{C}_{U}$ if and only if $O^{(\sigma)} U_{\phi} O^{(\tilde{\sigma})} \in \mathcal{C}_{U}$, for all permutations $\sigma, \tilde{\sigma}$. It may be that a design principle is on-site homogeneous only with respect to a sublattice $X^{\prime} \subset X$. In this case we simply treat $X^{\prime}$ as the spatial lattice and add the finite set $X / X^{\prime}$ to the $\mathrm{d}_{\mathrm{F}}$ internal degrees of freedom. Comparing with Sec. III A, on-site homogeneous design principles are analogous to the set of Hamiltonians $H^{\prime}$ which are stoquastic in an on-site homogeneous basis - any qudit permutation operator has non-negative entries in this basis, like the imaginary time evolution $e^{-\beta H^{\prime}}$.

With these two notions of locality and homogeneity in design principles, we now define the DQMC analog of locally stoquastic Hamiltonians (see Sec. III).

Definition (locally sign-free DQMC). Given a local fermion-boson Hamiltonian $H$, we say that $H$ allows for a locally sign-free DQMC simulation, if there exists a local unitary $U$, such that $H^{\prime}=U H U^{\dagger}$ has a local DQMC representation (17), which is termwise sign-free due to an on-site homogeneous design principle.

Note that the DQMC representation (17) is not of the Hamiltonian but of the partition function, and clearly $Z^{\prime}=$ $\operatorname{Tr}\left(e^{-\beta H^{\prime}}\right)=\operatorname{Tr}\left(e^{-\beta H}\right)=Z$. What the above definition entails is that it is $H^{\prime}$, rather than $H$, from which the DQMC data $S_{\phi}, h_{\phi(\tau)}$ are obtained, as described in Sec. V A. These data are then assumed to be termwise sign-free due to an on-site homogeneous design principle. The local unitary $U$ appearing in the above definition is generally fermionic [106]: it can be written as a finite-time evolution $U=\mathrm{TO} e^{-i \int_{0}^{1} \tilde{H}(t) d t}$, where $\tilde{H}$ is a local fermion-boson Hamiltonian, which is either piecewise-constant or smooth as a function of $t$; cf. Sec. III A.

\section{Example: Time-reversal design principle}

To demonstrate the above definitions in a concrete setting, consider the time-reversal design principle, defined by an antiunitary operator $\mathrm{T}$ acting on the single-fermion Hilbert space $\mathcal{H}_{1 \mathrm{~F}} \cong \mathbb{C}^{|X|} \otimes \mathbb{C}^{\mathrm{d}_{\mathrm{F}}}$, such that $\mathrm{T}^{2}=-I$. The set $\mathcal{C}_{h}$ contains all $\mathrm{T}$-invariant matrices, $\left[\mathrm{T}, h_{\phi(\tau)}\right]=0$. It follows that [T, $\left.U_{\phi}\right]=0$, so that $\mathcal{C}_{U}=\mathcal{C}_{h}$ in this case, and this implies $\operatorname{Det}\left(I+U_{\phi}\right) \geqslant 0[28,103]$.

A sufficient condition on $\mathrm{T}$ that guarantees that the design principle it defines is on-site homogenous is that it is of the form $\mathrm{T}_{0}=I_{|X|} \otimes \mathrm{t}$, where $I_{|X|}$ is the identity matrix on $\mathbb{C}^{|X|}$, and $\mathrm{t}$ is an antiunitary on $\mathbb{C}^{\mathrm{d}_{\mathrm{F}}}$ that squares to $-I_{\mathrm{d}_{F}}$. Equivalently, $\mathrm{T}$ is block diagonal, with identical blocks $t$ corresponding to the lattice sites $\mathbf{x} \in X$. It is then clear that the permutation matrices $O^{(\sigma)}$ defined in Eq. (20) commute with $\mathrm{T}$, so $O^{(\sigma)} \in \mathcal{C}_{U}$ for all $\sigma \in S_{X}$. Note that the design principle $\mathrm{T}$ may correspond to a physical time-reversal $\mathcal{T}$, discussed in Sec. IV, only if it is on-site homogenous, which is why we distinguish the two in our notation.

Additionally, if the operator $\mathrm{T}$ is $r_{\mathrm{T}}$-local with some range $r_{\mathrm{T}} \geqslant 0$, then any local $h_{\phi(\tau)}$ which is sign-free due to $\mathrm{T}$ can be made termwise sign-free. Indeed, if $\left[\mathrm{T}, h_{\phi(\tau)}\right]=0$ then

$$
\begin{aligned}
h_{\phi(\tau)} & =\frac{1}{2}\left(h_{\phi(\tau)}+\mathrm{T} h_{\phi(\tau)} \mathrm{T}^{-1}\right) \\
& =\sum_{\mathbf{x}} \frac{1}{2}\left(h_{\phi(\tau) ; \mathbf{x}}+\mathrm{T} h_{\phi(\tau) ; \mathbf{x}} \mathrm{T}^{-1}\right) \\
& =\sum_{\mathbf{x}} \tilde{h}_{\phi(\tau) ; \mathbf{x}},
\end{aligned}
$$

where $\tilde{h}_{\phi(\tau) ; \mathbf{x}}$ is now supported on a disk of radius $r+2 r_{\mathbf{T}}$ and commutes with $\mathrm{T}$, for all $\mathbf{x}$. We see that the specific notion of $r_{\mathrm{T}}$-locality coincides with the general notion of "termwise 
sign-free". In particular, $\mathrm{T}=\mathrm{T}_{0}$ has a range $r_{\mathrm{T}}=0$, and can therefore be applied termwise.

The above statements imply that if $\mathrm{T}=u \mathrm{~T}_{0} u^{\dagger}$, where $u$ is a single-fermion local unitary, and $H$ has a local DQMC representation which is sign-free due to $\mathrm{T}$, then $H$ allows for a locally sign-free DQMC simulation. Indeed, extending $u$ to a many-body local unitary $U$, we see that $H^{\prime}=U H U^{\dagger}$ admits a local DQMC representation where $\left[\mathrm{T}_{0}, h_{\phi(\tau)}^{\prime}\right]=0$. Since $\mathrm{T}_{0}$ is on-site homogenous, and $h_{\phi(\tau)}^{\prime}$ can be assumed termwise sign-free [see Eq. (21)], we have the desired result. As demonstrated in Appendix F, much of the above analysis carries over to other known design principles.

All realizations of T presented in Ref. [103] in the context of generalized Hubbard models, and in Ref. [17] in the context of quantum critical metals, have the on-site homogeneous form $T_{0}$, and therefore correspond to locally sign-free DQMC simulations.

We now consider a few specific time-reversal design principles $\mathrm{T}$. The physical spin-1/2 time reversal $\mathrm{T}=\mathcal{T}^{(1 / 2)}$, where $\mathcal{T}_{(\mathbf{x}, \alpha),\left(\mathbf{x}^{\prime}, \alpha^{\prime}\right)}^{(1 / 2)}=\delta_{\mathbf{x}, \mathbf{x}^{\prime}} \varepsilon_{\alpha \alpha^{\prime}} \mathcal{K}$, and $\alpha, \alpha^{\prime} \in\{\uparrow, \downarrow\}$ correspond to up and down spin components, is an on-site homogeneous design principle, which accounts for the absence of signs in the attractive Hubbard model [103]. The composition $T=$ $\mathcal{M} \mathcal{T}^{(1 / 2)}$ of $\mathcal{T}^{(1 / 2)}$ with a modulo 2 translation, $\mathcal{M}_{(\mathbf{x}, \alpha),\left(\mathbf{x}^{\prime}, \alpha^{\prime}\right)}=$ $\delta_{(-1)^{x},(-1)^{x^{\prime}+1}} \delta_{x_{e}, x_{e}^{\prime}} \delta_{y, y^{\prime}} \delta_{\alpha, \alpha^{\prime}}$, where $x_{e}=2\lfloor x / 2\rfloor$ is the even part of $x$, is an on-site homogeneous design principle with respect to the sublattice $X^{\prime}=\left\{\left(2 x_{1}, x_{2}\right): \mathbf{x} \in X\right\}$, but not with respect to $X$. On the other hand, the composition $T=\mathcal{P}^{(0)} \mathcal{T}^{(1 / 2)}$ of $\mathcal{T}^{(1 / 2)}$ with a spin-less reflection (or parity) $\mathcal{P}_{(\mathbf{x}, \alpha),\left(\mathbf{x}^{\prime}, \alpha^{\prime}\right)}^{(0)}=$ $\delta_{x,-x^{\prime}} \delta_{y, y^{\prime}} \delta_{\alpha \alpha^{\prime}}$, is not on-site homogeneous with respect to any sublattice.

The latter example is clearly nonlocal, and we use it to demonstrate the necessity of locality in design principles. As discussed in Sec. IV, the breaking of $\mathcal{P}$ and $\mathcal{T}$ down to $\mathcal{P} \mathcal{T}$ actually defines the notion of chirality, and therefore $\mathcal{P} \mathcal{T}$ is a natural symmetry in chiral topological matter. Accordingly, the design principle $T=\mathcal{P}^{(0)} \mathcal{T}^{(1 / 2)}$ applies to a class of models for chiral topological phases; see Appendix E. This seems to allow, from the naive algebraic perspective, for a sign-free DQMC simulation of certain chiral topological phases. However, the weights $p(\phi)$ will be non-negative only for bosonic configurations $\phi$ which are invariant under $T=\mathcal{P}^{(0)} \mathcal{T}^{(1 / 2)}$. Restricting the $\phi$ integration in Eq. (17) to such configurations leads to nonlocal interactions between fermions $\psi$, coupling the points $(x, y)$ and $(-x, y)$. These interactions effectively fold the nonlocal chiral system into a local nonchiral system of half of space; see Fig. 5. Thus, $T=\mathcal{P}^{(0)} \mathcal{T}^{(1 / 2)}$ does not allow for sign-free DQMC simulations of chiral topological matter.

\section{Sign-free geometric manipulations in DQMC}

Let $Z$ be a partition function in a local DQMC form (17), on the discrete torus $X=\mathbb{Z}_{N_{x}} \times \mathbb{Z}_{N_{y}}$ and imaginary time circle $S_{\beta}^{1}=\mathbb{R} / \beta \mathbb{Z}$, which is termwise sign-free due to an on-site homogenous design principle. In this section we show that it is possible to cut $X$ to the cylinder $C$, and subsequently introduce a screw dislocation in the space-time $C \times S_{\beta}^{1}$, which corresponds to the momentum polarization (3), while maintaining the DQMC weights $p(\phi)$ non-negative. (a)

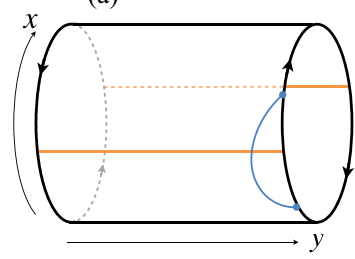

(c)

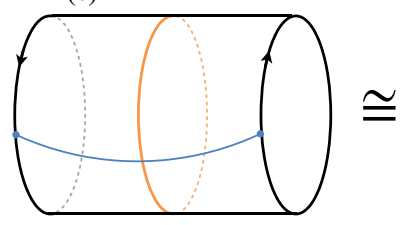

FIG. 5. $\mathcal{P} \mathcal{T}$ symmetry as a "nonlocal design principle" for chiral topological matter. (a, c) $\mathcal{P} \mathcal{T}$ symmetry, where $\mathcal{P}$ is a reflection (with respect to the orange lines) and $\mathcal{P} \mathcal{T}$ is an on-site time-reversal, is a natural symmetry in chiral topological phases. If $(\mathcal{P} \mathcal{T})^{2}=-I$, as is the case when $\mathcal{P}=\mathcal{P}^{(0)}$ is spin-less and $\mathcal{T}=\mathcal{T}^{(1 / 2)}$ is spin-full, it implies the non-negativity of fermionic determinants. Nevertheless, as $\mathcal{P} \mathcal{T}$ is nonlocal, it allows only for QMC simulations with $\mathcal{P} \mathcal{T}$ invariant bosonic fields, which mediate nonlocal interactions (blue lines) between fermions. Arrows indicate the chirality of boundary degrees of freedom. (b, d) Such nonlocal interactions effectively fold the system into a nonchiral locally interacting system supported on half the cylinder, where $\mathcal{P} \mathcal{T}$ acts as an on-site time reversal. In particular, the boundary degrees of freedom are now nonchiral. Thus, $\mathcal{P} \mathcal{T}$ does not allow for sign-free QMC simulations of chiral topological matter. More generally, fermionic design principles must be local in order to allow for sign-free DQMC simulations of local Hamiltonians.

\section{Introducing spatial boundaries}

Given a translation $T^{\mathbf{d}}(\mathbf{d} \in X)$, we can cut the torus $X$ along a line $l$ parallel to $\mathbf{d}$, and obtain a cylinder $C$ where $T^{\mathbf{d}}$ acts as a translation within each boundary component, as in Sec. III. Given the DQMC representation (17) on $X$, the corresponding representation on $C$ is obtained by eliminating all local terms $S_{\phi ; \tau, \mathbf{x}}, h_{\phi(\tau) ; \mathbf{x}}$ whose support overlaps $l$, as in Fig. 3. This procedure may render $S_{\phi}, h_{\phi(\tau)}$ independent of certain degrees of freedom $\phi(\mathbf{x}, \tau), \psi(\mathbf{x}, \tau)$, with $\mathbf{x}$ within a range $r$ of $l$, in which case we simply remove such degrees of freedom from the functional integral (17). ${ }^{7}$ Since $S_{\phi ; \tau, \mathbf{x}}, h_{\phi(\tau) ; \mathbf{x}}$ obey the design principle for every $\mathbf{x}, \tau$, the resulting $S_{\phi}, h_{\phi(\tau)}$ still obey the design principle and the weights $p(\phi)$ remain real and non-negative.

\section{Introducing a screw dislocation in space-time}

Let us now restrict attention to $\mathbf{d}=(1,0)$, and make contact with the momentum polarization (3). Given a partition function on the space-time $C \times S_{\beta}^{1}$, consider twisting the boundary conditions in the time direction,

$$
\phi_{\tau+\beta, x, y}=\phi_{\tau, x-\lambda \Theta(y), y}, \quad \psi_{\tau+\beta, x, y}=-\psi_{\tau, x-\lambda \Theta(y), y} .
$$

\footnotetext{
${ }^{7}$ For $r_{0}$-local $\phi$, which is defined on links, plaquettes, etc., we also remove from the functional $\phi$ integration those links, plaquettes, etc. which overlap $l$.
} 
Note that $\lambda \in \mathbb{Z}_{N_{x}}$, since $x \in \mathbb{Z}_{N_{x}}$. In particular, the full twist $\lambda=N_{x}$ is equivalent to the untwisted case $\lambda=0$, which is equivalent to the statement that the modular parameter of the torus is defined mod 1 (see, e.g., example 8.2 of Ref. [107]). The case $\lambda=0$ gives the standard boundary conditions, where the partition function is, in Hamiltonian terms, just $Z=$ $\operatorname{Tr}\left(e^{-\beta H}\right)$. In this case $Z>0$ since $H$ is Hermitian, though its QMC representation $Z=\sum_{\phi} p(\phi)$ will generically involve complex valued weights $p$. The twisted case $\lambda=1$ includes the insertion of the half-translation operator

$$
\tilde{Z}=\operatorname{Tr}\left(T_{R} e^{-\beta H}\right)
$$

which appears in the momentum polarization (3). Since $T_{R}$ is unitary rather than hermitian, $\tilde{Z}$ itself will generically be complex. However, we make the following claim:

Claim: If $Z$ has a local DQMC representation (17), which is termwise sign-free due to an on-site homogeneous design principle, then $\tilde{Z}$ also has a sign-free QMC representation: $\tilde{Z}=\sum_{\phi} \tilde{p}(\phi)$, with $\tilde{p}(\phi) \geqslant 0$. In particular, $\tilde{Z} \geqslant 0$.

Proof of the claim is provided below. It revolves around two physical points: (i) For the boson $\phi$, we use only the fact that all boundary conditions, and those in Eq. (22) in particular, are locally invisible. (ii) For the fermion $\psi$, the local invisibility of boundary conditions does not suffice, and the important point is that translations do not act on internal degrees of freedom, and therefore correspond to permutations of the lattice sites. The same holds for the half translation $T_{R}$. This distinguishes translations from internal symmetries, as well as from all other spatial symmetries, which involve point group elements, and generically act nontrivially on internal degrees of freedom. For example, a $C_{4}$ rotation will act nontrivially on spin-full fermions.

Proof. We first consider the fermionic part of the Boltzmann weight, $\operatorname{Det}\left(I+U_{\phi}\right)$. The Hamiltonian $h_{\phi(\tau)}$ depends on the values of $\phi$ at a single time slice $\tau$ and is therefore unaffected by the twist in bosonic boundary conditions. It follows that $U_{\phi}$ is independent of the twist in bosonic boundary conditions. On the other hand, the fermionic boundary conditions in (22) correspond to a change of the time evolution operator $U_{\phi} \mapsto T_{R} U_{\phi}$, in analogy with (23). Since the design principle $\mathcal{C}_{U}$ is assumed to be on-site homogeneous, and $T_{R}=O^{(\sigma)}$ is a permutation operator, with $\sigma:(x, y) \mapsto(x+\Theta(y), y)$, we have $T_{R} U_{\phi} \in \mathcal{C}_{U}$, and $\operatorname{Det}\left(I+T_{R} U_{\phi}\right) \geqslant 0$.

Let us now consider the bosonic part of the Boltzmann weight $e^{-S_{\phi}}$, where each of the local terms $S_{\phi ; \tau, \mathbf{x}}$ is manifestly real valued for all $\phi$. We assume that the imaginary time circle $S_{\beta}^{1}$ is discretized, such that the total number of spacetime points $(\tau, \mathbf{x})=u \in U$ is finite. Such a discretization is common in DQMC algorithms [19,21], and the continuum case can be obtained by taking the appropriate limit. The term $S_{\phi ; \tau, \mathbf{x}}$ can then be written as a composition $f \circ g_{V}$, where $f$ is a real-valued function, and $g_{V}:\left(\phi_{u}\right)_{u \in U} \mapsto\left(\phi_{u}\right)_{u \in V}$ chooses the values of $\phi$ on which $S_{\phi ; \tau, \mathbf{x}}$ depends, where $V \subset U$ is the support of $S_{\phi ; \tau, \mathbf{x}}$. The bosonic boundary conditions (22) then amount to a modification of the support $V \mapsto V_{\lambda}$, as depicted in Fig. 6, but not of the function $f$, which remains real valued. In particular, for $\lambda=1$ we have $S_{\phi ; \tau, \mathbf{x}} \mapsto \tilde{S}_{\phi ; \tau, \mathbf{x}}=f \circ g_{V_{1}}$, and $S_{\phi} \mapsto \tilde{S}_{\phi}=\sum_{\tau, \mathbf{x}} \tilde{S}_{\phi ; \tau, \mathbf{x}} \in \mathbb{R}$.

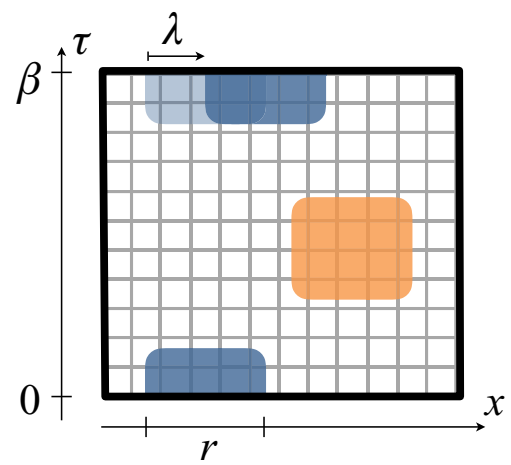

FIG. 6. Implementing the bosonic boundary conditions (22). The lattice lies in the $x$ - $\tau$ plane, at $y>0$ where the boundary conditions are nontrivial. The orange area marks the support, of diameter $r$, of a local term $S_{\phi ; \tau, \mathbf{x}}$ which is unaffected by the boundary conditions. Blue areas correspond to the support of a local term which is affected by the boundary conditions, with pale blue indicating the untwisted case $\lambda=0$.

Combining the above conclusions for the bosonic and fermionic parts of $\tilde{p}(\phi)=e^{-\tilde{S}_{\phi}} \operatorname{Det}\left(I+T_{R} U_{\phi}\right)$, we find that $\tilde{p}(\phi) \geqslant 0$ for all $\phi$.

\section{EXCLUDING SIGN-FREE DQMC FOR CHIRAL TOPOLOGICAL MATTER}

We are now ready to demonstrate the existence of an intrinsic sign problem in chiral topological matter comprised of bosons and fermions, using the machinery of Secs. III-V.

Let $H$ be a gapped local fermion-boson Hamiltonian on the discrete torus, which allows for a locally sign-free DQMC simulation. Unpacking the definition, this means that $H^{\prime}=$ $U H U^{\dagger}$ has a local DQMC representation which is termwise sign-free due to an on-site homogeneous design principle. As shown in Sec. VD, this implies that $\tilde{Z}^{\prime}:=\operatorname{Tr}\left(T_{R} e^{-\beta H^{\prime}}\right)$, written on the cylinder, also has a local DQMC representation, obeying a local and on-site design principle, and as a result, $\tilde{Z}^{\prime}>0$. Now, as shown in Sec. III, the positivity of $\tilde{Z}^{\prime}$ implies $\theta_{a}=e^{2 \pi i c / 24}$ for some anyon $a$. We therefore have the fermionic version of Result 1 ,

Result $1 F$. If a local fermion-boson Hamiltonian $H$, which is in a chiral topological phase of matter, allows for a locally sign-free DQMC simulation, then one of the corresponding topological spins satisfies $\theta_{a}=e^{2 \pi i c / 24}$. Equivalently, a chiral topological phase of matter where $e^{2 \pi i c / 24}$ is not the topological spin of some anyon, i.e., $e^{2 \pi i c / 24} \notin\left\{\theta_{a}\right\}$, admits no local fermion-boson Hamiltonians for which locally sign-free DQMC simulation is possible.

As shown in Sec. IV, the positivity of $\tilde{Z}^{\prime}$ implies $\theta_{a}=$ $e^{2 \pi i c / 24}$ for some anyon $a$, even if chirality appears only spontaneously. We therefore obtain the fermionic version of Result 2:

Result $2 F$. If a local fermion-boson Hamiltonian $H$, which is in a spontaneously chiral topological phase of matter, allows for a locally sign-free DQMC simulation, then one of the corresponding topological spins satisfies $\theta_{a}=e^{2 \pi i c / 24}$. Equivalently, a spontaneously chiral topological phase of matter where $e^{2 \pi i c / 24}$ is not the topological spin of some anyon, i.e., 
$e^{2 \pi i c / 24} \notin\left\{\theta_{a}\right\}$, admits no local fermion-boson Hamiltonians which allow for a locally sign-free DQMC simulation.

In stating these results, we do not restrict to fermionic phases, because bosonic phases may admit a fermionic description, for which DQMC is of interest. When a bosonic phase admits a fermionic description, the bosonic field $\phi$ in Eq. (17) will contain a $\mathbb{Z}_{2}$ gauge field that couples to the fermion parity $(-1)^{N_{f}}$ of $\psi$. An important series of examples is given by the non-Abelian Kitaev spin liquids, which admit a description in terms of gapped Majorana fermions with an odd Chern number $v$, coupled to a $\mathbb{Z}_{2}$ gauge field [46]. As described in Table I, the criterion $e^{2 \pi i c / 24} \notin\left\{\theta_{a}\right\}$ applies to the Kitaev spin liquid, for all $v \in 2 \mathbb{Z}-1$. Result 1 then excludes the possibility of locally stoquastic Hamiltonians for the microscopic description in terms of spins, while Result $1 \mathrm{~F}$ excludes the possibility of locally sign-free DQMC simulations in the emergent fermionic description.

\section{CONJECTURES: BEYOND CHIRAL MATTER}

In Secs. III-VI we established a criterion for the existence of intrinsic sign problems in chiral topological matter: if $e^{2 \pi i c / 24} \notin\left\{\theta_{a}\right\}$, or equivalently $1 \notin \operatorname{Spec}(\mathbf{T})$ (see Result $\left.1^{\prime}\right)$, then an intrinsic sign problem exists. Even if taken at face value, this criterion never applies to nonchiral bosonic topological phases, where $c=0$, due to the vacuum topological spin $1 \in\left\{\theta_{a}\right\}$. The same statement applies to all bosonic phases with $c \in 24 \mathbb{Z}$. In this section we propose a refined criterion for intrinsic sign problems in topological matter, which nontrivially applies to both chiral and nonchiral cases, and also unifies the results of this paper with those obtained by other means in our parallel work [38].

Reference [94] proposed the "universal wave-function overlap" method for characterizing topological order from any basis $\{|i\rangle\}$ for the ground-state subspace of a local gapped Hamiltonian $H$ on the torus $X$. The method is based on the conjecture

$$
\left\langle i\left|\mathbf{T}_{\mathrm{m}}\right| j\right\rangle=e^{-\alpha_{\mathbf{T}} A+o\left(A^{-1}\right)} \mathbf{T}_{i j},
$$

where $A$ is the area of the torus, $\alpha_{\mathbf{T}}$ is a nonuniversal complex number with non-negative real part, the microscopic Dehn-twist operator $\mathbf{T}_{\mathrm{m}}$ implements the Dehn twist $(x, y) \mapsto$ $(x+y, y)$ on the Hilbert space, and $\mathbf{T}_{i j}$ are the entries of the topological $\mathbf{T}$ matrix that characterizes the phase of $H$, in the basis $\{|i\rangle\}$. The same statement applies to any element $\mathbf{M}$ of the mapping class group of the torus, isomorphic to $S L(2, \mathbb{Z})$, with $\mathbf{M}$ in place of $\mathbf{T}$ in Eq. (24). The nonuniversal exponential suppression of the overlap is expected because $\mathbf{M}_{\mathrm{m}}$ will not generically map the ground-state subspace to itself, but if $\mathbf{M}_{\mathrm{m}}$ happens to be a symmetry of $H$, then $\alpha_{\mathbf{M}}=0[80,108]$. Though we are not aware of a general analytic derivation of Eq. (24), it was verified analytically and numerically in a large number of examples in Refs. [94,98,109-111], for Hamiltonians in both chiral and nonchiral phases.

Note the close analogy between Eq. (24) and the momentum polarization (3), where the microscopic Dehn-twist $\mathbf{T}_{\mathrm{m}}$ on the torus and the half translation $T_{R}$ on the cylinder play a similar role, and nonuniversal extensive contributions are followed by subextensive universal data. To make this analogy clearer, and make contact with the analysis of Secs. III and VI, we consider the object $Z_{\mathbf{T}}=\operatorname{Tr}\left(\mathbf{T}_{\mathrm{m}} e^{-\beta H}\right)$, which satisfies

$$
Z_{\mathbf{T}}=Z e^{-\alpha_{\mathbf{T}} A+o\left(A^{-1}\right)} \operatorname{Tr}(\mathbf{T}),
$$

and can be interpreted as either the (unnormalized) thermal expectation value of $\mathbf{T}_{\mathrm{m}}$, or the partition function on a spacetime twisted by $\mathbf{T}$, in analogy with Sec. II C. Equation (25) is valid for temperatures $\Delta E \ll 1 / \beta \ll E_{\mathrm{g}}$, much lower than the bulk gap $E_{\mathrm{g}}$ and much higher than any finite-size splitting in the ground-state subspace, $\Delta E=o\left(A^{-1}\right)$.

Just like $T_{R}$, the operator $\mathbf{T}_{\mathrm{m}}$ acts as a permutation of the lattice sites. Therefore, following Secs. III and VI, if $H$ is either locally stoquastic, or admits a locally sign-free DQMC simulation, then $\operatorname{Tr}(\mathbf{T}) \geqslant 0$. In terms of $c$ and $\left\{\theta_{a}\right\}$, this implies $e^{-2 \pi i c / 24} \sum_{a} \theta_{a}=\operatorname{Tr}(\mathbf{T}) \geqslant 0$, where the sum runs over all topological spins.

The last statement applies to both bosonic and fermionic Hamiltonians. For bosonic Hamiltonians, it can be strengthened by means of the Frobenius-Perron theorem. If $H^{\prime}=U H U^{\dagger}$ is stoquastic in the on-site basis $|s\rangle$, Hermitian, and has a degenerate ground-state subspace, then this subspace can be spanned by an orthonormal basis $\left|i^{\prime}\right\rangle$ with positive entries in the on-site basis, $\left\langle s \mid i^{\prime}\right\rangle \geqslant 0$; see, e.g., Ref. [38]. This implies that

$$
0 \leqslant\left\langle i^{\prime}\left|\mathbf{T}_{\mathrm{m}}\right| j^{\prime}\right\rangle=e^{-\alpha_{\mathbf{T}}^{\prime} A+o\left(A^{-1}\right)} \mathbf{T}_{i^{\prime} j^{\prime}},
$$

where $\alpha_{\mathbf{T}}^{\prime}$ is generally different from $\alpha_{\mathbf{T}}$, but the matrix $\mathbf{T}_{i^{\prime} j^{\prime}}$ has the same spectrum as $\mathbf{T}_{i j}$ in Eq. (24). This is a stronger form of (25), which implies $\mathbf{T}_{i^{\prime} j^{\prime}} \geqslant 0$. Since $\mathbf{T}_{i^{\prime} j^{\prime}}$ is also unitary, it is a permutation matrix, $\mathbf{T}_{i^{\prime} j^{\prime}}=\delta_{i^{\prime}, \sigma\left(j^{\prime}\right)}$ for some $\sigma \in S_{N}$, where $N$ is the number of ground states. In turn, this implies that the spectrum of $\mathbf{T}$ is a disjoint union of complete sets of roots of unity,

$$
\left\{\theta_{a} e^{-2 \pi i c / 24}\right\}_{a=1}^{N}=\operatorname{Spec}(\mathbf{T})=\bigcup_{k=1}^{K} R_{n_{k}},
$$

where $R_{n_{k}}$ is the set of $n_{k}$ th roots of unity, $n_{k}, K \in \mathbb{N}$, and $\sum_{k=1}^{K} n_{k}=N$. Therefore,

Conjecture 1. A bosonic topological phase of matter where $\left\{\theta_{a} e^{-2 \pi i c / 24}\right\}$ is not a disjoint union of complete sets of roots of unity admits no local Hamiltonians which are locally stoquastic.

In particular, this implies an intrinsic sign problem whenever $1 \notin\left\{\theta_{a} e^{-2 \pi i c / 24}\right\}$, thus generalizing Result 1 . Moreover, the above statement applies nontrivially to phases with $c \in 24 \mathbb{Z}$. In particular, for nonchiral phases, where $c=0$, it reduces to the result established in Ref. [38], thus generalizing it as well. The simplest example for a nonchiral phase with an intrinsic sign problem is the doubled semion phase, where $\left\{\theta_{a}\right\}=\{1, i,-i, 1\}[37]$.

Though we are currently unaware of an analog of the Frobenius-Perron theorem that applies to DQMC, we expect that an analogous result can be established for fermionic Hamiltonians.

Conjecture 1F. A topological phase of matter where $\left\{\theta_{a} e^{-2 \pi i c / 24}\right\}$ is not a disjoint union of complete sets of roots of unity, admits no local fermion-boson Hamiltonians for which locally sign-free DQMC simulation is possible. 
The above conjectures suggest a substantial improvement over the criterion $e^{2 \pi i c / 24} \notin\left\{\theta_{a}\right\}$. To demonstrate this, we go back to the $1 / q$ Laughlin phases and SU(2) $)_{k}$ Chern-Simons theories considered in Table I. We find a conjectured intrinsic sign problem in all of the first 1000 bosonic Laughlin phases $(q$ even), fermionic Laughlin phases ( $q$ odd), and SU(2) $k$ ChernSimons theories. In particular, we note that the prototypical $1 / 3$ Laughlin phase is not captured by the criterion $e^{2 \pi i c / 24} \notin$ $\left\{\theta_{a}\right\}$, but is conjectured to be intrinsically sign-problematic.

\section{DISCUSSION AND OUTLOOK}

In this paper we established the existence of intrinsic sign problems in a broad class of chiral topological phases, namely, those where $e^{2 \pi i c / 24}$ does not happen to be the topological spin of an anyon. Since these intrinsic sign problems persist even when chirality, or time-reversal symmetry breaking, appears spontaneously, they are rooted in the macroscopic and observable data $c,\left\{\theta_{a}\right\}$, rather than the microscopic absence (or presence) of time-reversal symmetry. Going beyond the simple setting of stoquastic Hamiltonians, we provided the first treatment of intrinsic sign problems in fermionic systems. In particular, we constructed a general framework which describes all DQMC algorithms and fermionic design principles that we are aware of, including the state-of-art design principles [18,22-24] which are only beginning to be used by practitioners. Owing to its generality, it is likely that our framework will apply to additional design principles which have not yet been discovered, insofar as they are applied locally. We also presented conjectures that strengthen our results and unify them with those obtained in Refs. [9,38], under a single criterion in terms of $c$ and $\left\{\theta_{a}\right\}$. These conjectures also imply intrinsic sign problems in many topological phases not covered by existing results.

Conceptually, our results show that the sign problem is not only a statement of computational complexity: it is, in fact, intimately connected with the physically observable properties of quantum matter. Such a connection has long been heuristically appreciated by QMC practitioners and is placed on a firm and quantitative footing by the discovery of intrinsic sign problems.

Despite the progress made here, our understanding of intrinsic sign problems is still in its infancy, and many open questions remain:

Quantum computation and intrinsic sign problems. Intrinsic sign problems relate the physics of topological phases to their computational complexity, in analogy with the classification of topological phases which enable universal quantum computation $[48,49]$. As we have seen, many phases of matter that are known to be universal for quantum computation are also intrinsically sign-problematic, supporting the paradigm of "quantum advantage" or "quantum supremacy" [50]. Determining whether intrinsic sign problems appear in all phases of matter which are universal for quantum computation is an interesting open problem. Additionally, we identified intrinsic sign problems in many topological phases which are not universal for quantum computation. The intermediate complexity of such phases between classical and quantum computation is another interesting direction for future work.
Unconventional superconductivity and intrinsic sign problems. As described in the introduction, a major motivation for the study of intrinsic sign problems comes from long-standing open problems in fermionic many-body systems, the nature of high temperature superconductivity in particular. It is currently believed that many high temperature superconductors, and the associated repulsive Hubbard models, are nonchiral $d$ wave superconductors [17,27], in which we did not identify an intrinsic sign problem. The optimistic possibility that the sign problem can in fact be cured in repulsive Hubbard models is therefore left open, though this has not yet been accomplished in the relevant regime of parameters, away from half filling, despite intense research efforts [26]. Nevertheless, the state of the art DMRG results of Ref. [27] does not exclude the possibility of a chiral $d$-wave superconductor $(\ell= \pm 2$ in Table I). In this case we do find an intrinsic sign problem, which would account for the notorious sign problems observed in repulsive Hubbard models. More speculatively, it is possible that the mere proximity of repulsive Hubbard models to a chiral $d$ wave phase stands behind their notorious sign problems. The possible effect of an intrinsic sign problem in a given phase on the larger phase diagram was recently studied in Ref. [112]. There is also evidence for chiral $d$-wave superconductivity in doped graphene and related materials [113,114], and our results therefore suggest the impossibility of sign-free QMC simulations of these. We believe that the study of intrinsic sign problems in the context of unconventional superconductivity is a promising direction for future work.

Nonlocality as a possible route to sign-free QMC. The intrinsic sign problems identified in this work add to existing evidence for the complexity of chiral topological phases - these do not admit local commuting projector Hamiltonians [54,115-117], nor do they admit local Hamiltonians with a PEPS state as an exact ground state [43,118-120].

Nevertheless, relaxing the locality requirement does lead to positive results for the simulation of chiral topological matter using commuting projectors or PEPS. First, commuting projector Hamiltonians can be obtained if the local bosonic or fermionic degrees of freedom are replaced by anyonic (and therefore nonlocal) excitations of an underlying chiral topological phase [121]. Second, chiral topological Hamiltonians can have a PEPS ground state if they include interactions (or hopping amplitudes) that slowly decay as a power law with distance.

One may therefore hope that sign-free QMC simulations of chiral topological matter can also be performed if the locality requirements made in Sec. $\mathrm{V}$ are similarly relaxed. Do such "weakly local" sign-free models exist?

Easing intrinsic sign problems. In this paper we proved the existence of an intrinsic sign problem in chiral topological phases of matter, but we did not quantify the severity of this sign problem, which is an important concept in both practical applications and theory of QMC. The severity of a sign problem is quantified by the smallness of the average sign of the QMC weights $p$ with respect to the distribution $|p|$, i.e., $\langle\operatorname{sgn}\rangle:=\sum p / \sum|p|$. Since $\langle\operatorname{sgn}\rangle$ can be viewed as the ratio of two partition functions, it obeys the generic scaling $\langle\operatorname{sgn}\rangle \sim e^{-\Delta \beta N}$, with $\Delta \geqslant 0$, as $\beta N \rightarrow \infty[2,10]$. A sign problem exists when $\Delta>0$, in which case QMC simulations require exponential computational resources, and this is what 
the intrinsic sign problem we identified implies for "most" chiral topological phases of matter. From the point of view of computational complexity, all that matters is whether $\Delta=0$ or $\Delta>0$, but for practical applications the value of $\Delta$ is very important; see, e.g., Ref. [113]. One may hope for a possible refinement of our results that provides a lower bound $\Delta_{0}>0$ for $\Delta$, but since we have studied topological phases of matter, we view this as unlikely. It may therefore be possible to obtain fine-tuned models and QMC methods that lead to a $\Delta$ small enough to be practically useful. More generally, it may be possible to search for such models and methods algorithmically, thus easing the intrinsic sign problem [10,122-124]. We also note that the results presented in this paper do not exclude approaches to the sign problem based on a modified or constrained Monte Carlo sampling [13,125,126], as well as machine-learning-aided QMC [127], and infinite-volume diagrammatic QMC [128].

Possible extensions. The chiral central charge appears only modulo 24 in our results. Nevertheless, the full value of $c$ is physically meaningful, as reviewed in the introduction. Does an intrinsic sign problem exist in all phases with $c \neq 0$ ? The results of Ref. [39] strongly suggest this.

The arguments of Ref. [38] and Sec. VII apply equally well to any element of the modular group, rather than just the topological $\mathbf{T}$ matrix, implying that the spectrum of all elements decomposes into full sets of roots of unity. This may imply a more restrictive constraint on the TFT data than conjectured in Sec. VII.

It is believed that all SPT phases can be characterized by universal complex phases acquired by their partition functions, when placed on certain nontrivial space-times [75,129-132], in analogy with Eq. (3). Loosely speaking, for an SPT with on-site symmetry group $G$, the relevant spacetime would be obtained by purely geometric manipulations as performed in this paper, along with a twisting of boundary conditions by elements $g \in G$. Since each $G$ acts on-site, we do not expect intrinsic sign problems whenever a nontrivial $g$ is required to detect the SPT [133-136]. Nevertheless, it may be possible to obtain weaker statements, constraining the possible bases in which a Hamiltonian in a $G$-SPT may be stoquastic. Such constraints may be more useful for designing sign-free models than the stronger intrinsic sign problems discussed in this paper. Similar questions arise in the context of topologically ordered phases, enriched by an on-site symmetry.

Finally, going beyond gapped topological phases, are there intrinsically sign-problematic phases which are not gapped, not topological, or both? It is the authors' hope that answers to some of these questions will shed new light on the quantum many-body problem.

\section{ACKNOWLEDGMENTS}

We thank Ryan Thorngren for participation in early stages of this work and feedback on the manuscript, and Snir Gazit for providing the much needed practitioner's point of view on DQMC and the sign problem. We also benefited from discussions with Ady Stern, Ari Turner, Ciarán Hickey, Erez Berg, Eyal Cornfeld, Eyal Leviatan, Johannes Stephan Hofmann, Michael Levin, Paul Wiegmann, and Raquel
Queiroz. This work was supported by the Israel Science Foundation (ISF, 2250/19), the Deutsche Forschungsgemeinschaft (DFG, German Research Foundation, CRC/Transregio 183, EI 519/7-1), and the European Research Council (ERC), under Project LEGOTOP and the European Union's Horizon 2020 research and innovation program (Grant Agreement No. 771537).

\section{APPENDIX A: FURTHER DETAILS REGARDING EQ. (3)}

This Appendix involves basic facts in CFT, which can be found in, e.g., Refs. [84,85].

\section{Definition of $h_{0}$ and ambiguities in its value}

A chiral topological phase of matter has a finitedimensional ground-state subspace on the spatial torus. A basis $\{|a\rangle\}_{a=1}^{N}$ for the torus ground-state subspace exists, such that each state $|a\rangle$ corresponds to a conformal family in the boundary CFT $[40,41,108]$, constructed over a primary with right- or left-moving conformal weights $h_{a}^{(l)}, h_{a}^{(r)} \geqslant 0$. The corresponding chiral and total conformal weights are then given by $h_{a}=h_{a}^{(l)}-h_{a}^{(r)}$ and $h_{a}^{+}=h_{a}^{(l)}+h_{a}^{(r)}$, respectively. The chiral and total central charges of the CFT are similarly defined in terms of the left- or right-moving central charges, $c=c^{(l)}-c^{(r)}$ and $c^{+}=c^{(l)}+c^{(r)}$.

When the torus is cut to a cylinder with finite circumference $L$, the ground-state degeneracy is lifted, generically leaving a unique ground state. The lowest energy eigenstates on the cylinder can also be labeled as $\{|a\rangle\}_{a=1}^{N}$. Each $|a\rangle$ corresponds to a nonuniversal choice of state in the conformal family labeled by $h_{a}^{(l)}, h_{a}^{(r)}$, which need not be the primary, as demonstrated explicitly in Appendix B.

If the boundary is described by an idealized CFT, all $|a\rangle$ s correspond to primaries, and the corresponding energies are given by $E_{a}=(4 \pi v / L)\left(h_{a}^{+}-c^{+} / 24\right)$, relative to the groundstate energy on the torus, where $v$ is the velocity of the CFT and $L$ is the circumference of the cylinder. These expressions receive exponentially small corrections of $O\left(L e^{-R / \xi}\right)$ and $O\left(R e^{-L / \xi}\right)$, where $\xi$ is the bulk correlation length and $R$ is the length of the cylinder [80]. The cylinder ground state then corresponds to the CFT ground state, the primary with minimal $h_{a}^{+}$.

More generally, each state $|a\rangle$ corresponds to either a primary or a descendent and has conformal weights $h_{a}^{(l)}+$ $n_{a}^{(l)}, h_{a}^{(r)}+n_{a}^{(r)}$, where $n_{a}^{(l)}, n_{a}^{(r)} \in \mathbb{N}_{0}$. The corresponding energies $E_{a}$ differ from the idealized $(4 \pi v / L)\left(h_{a}^{+}-c^{+} / 24\right)$, and the choice of conformal family $a_{0}$ with minimal $E_{a_{0}}$ is nonuniversal. In terms of $n_{a}=n_{a}^{(l)}-n_{a}^{(r)}$, we then define $h_{0}:=h_{a_{0}}+n_{a_{0}}$, the chiral conformal weight associated with the cylinder ground state $\left|a_{0}\right\rangle$. The value of $h_{0}$ therefore carries two ambiguities: a choice of conformal family $a_{0} \in\{a\}$, and the choice of a state in the conformal family, $n_{a_{0}} \in \mathbb{N}_{0}$. As described in Sec. II B, the only universal statement is $\theta_{0}=$ $e^{2 \pi i h_{0}} \in\left\{\theta_{a}\right\}$, where $\theta_{a}=e^{2 \pi i h_{a}}$ are the topological spins of bulk anyons.

The result of Ref. [40] for the momentum polarization is given in terms of the low-lying cylinder eigenstates $|a\rangle$,

$$
\left\langle a\left|T_{R}\right| a\right\rangle=\exp \left[\alpha N_{x}+\frac{2 \pi i}{N_{x}}\left(h_{a}-\frac{c}{24}\right)+o\left(N_{x}^{-1}\right)\right],
$$


where the lattice spacing is set to $1, N_{x}=L$. It follows that the thermal expectation value $\tilde{Z} / Z=\operatorname{Tr}\left(T_{R} e^{-\beta H}\right) / Z$ is equal to $\exp \left[\alpha N_{x}+\frac{2 \pi i}{N_{x}}\left(h_{0}-\frac{c}{24}\right)+o\left(N_{x}^{-1}\right)\right]$, if the temperature $\beta^{-1}$ is much lower than the boundary energy differences $\sim N_{x}^{-1}$, namely, $\beta^{-1}=o\left(N_{x}^{-1}\right)$, as described in Sec. II.

\section{The value of $\boldsymbol{h}_{\mathbf{0}}$ in fermionic phases of matter}

Fermionic topological phases are microscopically comprised of fermions (and possibly bosons) and have the fermion parity $(-1)^{N_{f}}$ as a global symmetry $[47,75,137,138]$. It is therefore useful to probe such phases with a background $\mathbb{Z}_{2}$ gauge field corresponding to $(-1)^{N_{f}}$ or a spin structure. For our purposes, this amounts to considering both periodic and antiperiodic boundary conditions around noncontractible cycles in space-time.

In the main text we were interested only in locally signfree QMC representations of thermal partition functions, and sign-free geometric manipulations that can be performed to these. We therefore restricted attention to thermal boundary conditions in the imaginary time direction (see Sec. V A) and to periodic boundary conditions around the spatial cylinder. These boundary conditions cannot generically be modified without introducing signs into the QMC weights.

Here we provide a fuller picture by considering the behavior of $h_{0}$ with both periodic and antiperiodic boundary conditions, in the closed $x$ direction of the spatial cylinder. Since $h_{0}$ is a ground-state property, the time direction is open and does not play a role.

For a fermionic chiral topological phase, the boundary CFT is also fermionic. The primary conformal weights $\left\{h_{a}\right\}$ then depend on the choice of boundary conditions (in the $x$ direction), and as a result, so will the set of topological spins $\left\{\theta_{a}\right\}$ in which $\theta_{0}=e^{2 \pi i h_{0}}$ is valued. In particular, the vacuum spin $\theta_{I}=0$ will not be included in $\left\{\theta_{a}\right\}$ for periodic boundary conditions, while for antiperiodic boundary conditions, both the vacuum $\theta_{I}=1$ and the spin $\theta_{\psi}=-1$ of the microscopic fermion will appear [41,84]. Note that $\theta_{\psi}$ does not correspond to an emergent fermion, as in, e.g., the toric code [139], and therefore does not imply an additional ground state on the torus.

As an example, consider the series of Laughlin phases at filling $1 / q$, with $q \in \mathbb{N}$, all of which have the chiral central charge $c=1$. First, for $q \in 2 \mathbb{N}$ the phase is bosonic, and we consider only periodic boundary conditions. The primary conformal weights are given by $h_{a}=a^{2} / 2 q$ [36,140], with $a \in \mathbb{N}_{0}$. The topological spins $\theta_{a}=e^{2 \pi i h_{a}}$ depend only on $a$ $\bmod q$, and the $q$ spins $\left\{\theta_{a}\right\}_{a=0}^{q-1}$ (appearing in Table I) correspond to the $q$ degenerate ground states on the torus. In particular, the vacuum spin $\theta_{I}=1$ is obtained for $a=0$.

For $q \in 2 \mathbb{N}-1$ the phase is fermionic, and we consider both periodic and antiperiodic boundary conditions. For periodic boundary conditions the weights are given by $h_{a}=$ $(a+1 / 2)^{2} / 2 q[36]$. As in the bosonic case, $\theta_{a}=e^{2 \pi i h_{a}}$ depend only on $a \bmod q$, with $\left\{\theta_{a}\right\}_{a=0}^{q-1}$ (appearing in Table I) corresponding to the $q$ degenerate ground states on the torus. Unlike the bosonic case, the vacuum $\operatorname{spin} \theta_{I}=1$ is not included in $\left\{\theta_{a}\right\}_{a=0}^{q-1}$. For antiperiodic boundary conditions, the weights are given by $h_{a}=a^{2} / 2 q$ as in the bosonic case [140].
The set $\left\{\theta_{a}\right\}_{a=0}^{q-1}$ again corresponds to the $q$ torus ground states, but now $\theta_{\psi}=\theta_{a=q}=-1$ is an additional topological spin that corresponds to the physical fermion $\psi$ [69].

The simplest fermionic Laughlin phase is given by $q=1$, and corresponds to a Chern insulator with Chern number $v=1[86,87]$, which is studied in detail in Appendix B. The Chern insulator has a unique ground state on the torus, and accordingly, there is a unique topological spin $\theta_{\sigma}=e^{2 \pi i(1 / 8)}$ for periodic boundary conditions on the cylinder, and two topological spins $\theta_{I}=1, \theta_{\psi}=-1$ for antiperiodic boundary conditions. Here $\psi$ corresponds the physical fermions from which the Chern insulator is comprised. The object carrying the spin $\theta_{\sigma}$ is the complex analog of the celebrated Majorana zero mode supported on vortices in the bulk of a $p+i p$ superconductor $[46,52]$.

\section{APPENDIX B: MOMENTUM POLARIZATION WITH NON-CFT BOUNDARIES}

As reviewed in Sec. II, the existing analytic derivation of Eq. (3) relies on the CFT description of the physical boundaries of the cylinder, and of the line $y=0$ where $T_{R}$ is discontinuous [40]. In this Appendix we perform an analytic and numerical study that shows that, at least for free fermions, the relevant CFT expressions and the resulting Eq. (3) hold even if the boundary is not described by an idealized CFT. We will, however, find a number of subtleties which have not been demonstrated in the literature, as already described below Eq. (2) and in Appendix A.

\section{CFT finite-size correction in non-CFT boundaries}

The main ingredient in the analytic derivation of Eq. (3) is the expression (2) for the finite-size correction to the momentum density in CFT [40]. In this Appendix we show that, at least in the noninteracting case, Eq. (2) remains valid, with $\theta_{0}=e^{2 \pi i h_{0}} \in\left\{\theta_{a}\right\}$, even when the boundary cannot be described by a CFT.

We will consider a Chern insulator, such as the prototypical Haldane model [86]. When the boundary degrees of freedom can be described by a CFT, they correspond to the Weyl fermion CFT, where $c= \pm 1$ and the primary conformal weights are $h_{\sigma}= \pm 1 / 8\left(h_{I}=0, h_{\psi}= \pm 1 / 2\right)$ for periodic (antiperiodic) boundary conditions, as described in Appendix A2. The sign corresponds to the two possible chiralities. More generally, on a lattice with spacing 1 , the boundary supports a complex fermion with an energy dispersion $\varepsilon_{k}$, where $k=k_{x}$ takes values in the Brillouin zone $\mathbb{R} / 2 \pi \mathbb{Z}$ for an infinite circumference $L=\infty$, or its discretization $(2 \pi / L) \mathbb{Z}_{L}\left[(2 \pi / L)\left(\mathbb{Z}_{L}+1 / 2\right)\right]$, for $L<\infty$ and periodic (antiperiodic) boundary conditions. The only requirement on $\varepsilon_{k}$ is that it be chiral, in the sense that it connects the two separated bulk energy bands. If the intersections $k_{l}$ and $k_{u}$ with the lower and upper bulk bands, respectively, satisfy $k_{l}<k_{u}$ $\left(k_{l}>k_{u}\right)$, we say that the boundary is right (left) moving, or has a positive (negative) chirality; see Fig. 7.

More generally, the dispersion will contain several dispersion branches $\left\{\varepsilon_{j, k}\right\}_{j=1}^{J}$, but since the momentum density is additive in $j$ we restrict attention to a single branch. Without loss of generality, we also fix the chemical potential $\mu=0$, 


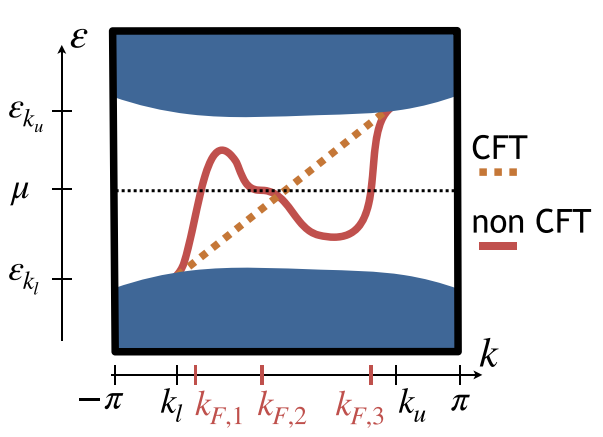

FIG. 7. Schematic band structure, energy $\varepsilon$ as a function of momentum $k=k_{x}$ in the periodic $x$ direction, of a Chern insulator on the cylinder. The figure shows the bulk energy bands (blue) and the chiral boundary dispersion, with two dispersion branches, on a single boundary component (orange and red curves). The opposite chirality branches on the second boundary component are not drawn. The momenta $k_{l}$ and $k_{u}$ correspond to the intersections of the boundary dispersion with the lower and upper bulk bands, respectively. Since $k_{u}>k_{l}$, both dispersion branches have a positive chirality. The orange line indicates the idealized linear dispersion with a single Fermi momentum $k_{F}=0$, which corresponds to the Weyl fermion CFT. The solid red curve corresponds to a more general chiral branch, with three Fermi momenta $k_{F, 1}, k_{F, 2}, k_{F, 3}$, where the dispersion around $k_{F, 2}$ takes a (nongeneric) nonlinear form. With periodic boundary conditions around the cylinder, both dispersion branches produce the same $L^{-2}$ correction to the momentum density in Eq. (B2), with a positive chirality + , up to a mod 1 ambiguity: $1 / 12 \mapsto 1 / 12+n, n \in \mathbb{N}$.

in which case the Fermi momentum $k_{F}$ satisfies $\varepsilon_{k_{F}}=0$. The value of $k_{F}$ plays an important role is the subsequent analysis.

The simplest dispersion that satisfies the above requirements is the linear one $\varepsilon_{k}=v\left(k-k_{F}\right)$. For $k_{F}=0$ this corresponds to the Weyl fermion CFT. The presence of $k_{F} \neq$ 0 corresponds to the addition of a chemical potential $v k_{F}$, which breaks the conformal symmetry. The generic form is $\varepsilon_{k}=v\left(k-k_{F}\right)+O\left(k-k_{F}\right)^{2}$. A nongeneric dispersion can take the form $\varepsilon_{k}=v_{3}\left(k-k_{F}\right)^{3}+O\left(k-k_{F}\right)^{4}$, and there may be several Fermi momenta if the dispersion is non monotonic; see Fig. 7.

In all cases the many-body ground-state momentum is given by summing the momenta of all filled single Fermion states $p(L)=\frac{1}{L} \sum_{\varepsilon_{k}<0} k$, where the sum runs over $k \in$ $(2 \pi / L) \mathbb{Z}_{L}$ such that $\varepsilon_{k}$ is negative and in the bulk energy gap. In order to obtain $p$ as a continuous function of $L$, we treat the bulk energy gap as a smooth cutoff $p(L)=\frac{1}{L} \sum_{\varepsilon_{k}<0} k C\left(\varepsilon_{k}\right)$, where the function $C(\varepsilon)$ goes to $1(0)$ fast enough as $\varepsilon$ goes to $0\left(\varepsilon_{k_{l}}\right.$ or $\left.\varepsilon_{k_{u}}\right) .{ }^{8}$ The cutoff $C$ represents the smooth delocalization of boundary eigenstates as their energy nears the bulk energy bands.

To obtain the $L$ dependence of $p(L)$, we will use the EulerMaclaurin formula

$$
\begin{aligned}
\sum_{n=n_{1}}^{n_{2}} f(n)= & \int_{n_{1}}^{n_{2}} f(x) d x+\frac{f\left(n_{2}\right)+f\left(n_{1}\right)}{2}+\frac{1}{6} \frac{f^{\prime}\left(n_{2}\right)-f^{\prime}\left(n_{1}\right)}{2 !} \\
& -\frac{1}{30} \frac{f^{\prime \prime \prime}\left(n_{2}\right)-f^{\prime \prime \prime}\left(n_{1}\right)}{4 !}+\mathrm{R},
\end{aligned}
$$

\footnotetext{
${ }^{8}$ It suffices that $C^{\prime}(\varepsilon)$ vanish at $\varepsilon=0, \varepsilon_{k_{l}}, \varepsilon_{k_{u}}$.
}

where the remainder satisfies $|\mathrm{R}| \leqslant \frac{2 \zeta(5)}{(2 \pi)^{5}} \int_{n_{1}}^{n_{2}}\left|f^{(5)}(x)\right| d x$. We begin by considering periodic boundary conditions, where we set $f(n)=\left(2 \pi n / L^{2}\right) C\left(\varepsilon_{2 \pi n / L}\right)$. Assuming a single, vanishing, Fermi momentum $k_{F}=0$, we set $\left(n_{1}, n_{2}\right)=(-\infty, 0)$ for positive chirality, and $\left(n_{1}, n_{2}\right)=(0, \infty)$ for negative chirality. Equation (B1) then gives

$$
p(L)=p(\infty) \pm \frac{2 \pi}{L^{2}} \frac{1}{12}+O\left(\frac{1}{L^{4}}\right), \quad \text { as } L \rightarrow \infty,
$$

where $p(\infty)=\int_{\varepsilon_{k}<0} k C\left(\varepsilon_{k}\right) d k / 2 \pi$ and $\pm=\operatorname{sgn}\left(k_{u}-k_{l}\right)$ is the chirality. The $1 / L^{2}$ correction in (B2) comes from $f^{\prime}(0)=$ $2 \pi / L^{2}$ in (B1). We see that the leading finite-size correction $h_{0}-c / 24$ is unchanged from its CFT value $h_{\sigma}-c / 24=$ $\pm 1 / 12$, even when a CFT description does not apply.

The case of a single nonzero Fermi momentum $k_{F} \neq 0$ is more interesting, as it demonstrates that the integer part of $h_{0}$ can change as a function of $L$ and $k_{F}$. The direct derivation of the end result from the Euler-Maclaurin formula is surprisingly lengthy, so we omit it and present a more direct route to the end result. To be concrete, assume a positive chirality and $k_{F}>0$. The Euler-Maclaurin formula leads to cutoff independent results, so we can restrict attention to cutoff functions $C\left(\varepsilon_{k}\right)$ which are identically 1 for $0<k<k_{F}$. Since these can serve as cutoff functions for the case $k_{F}=0$ as well, we can deduce the $k_{F} \neq 0$ momentum density $p\left(L, k_{F}\right)$ from the $k_{F}=0$ momentum density $p(L)$,

$$
\begin{aligned}
p\left(L, k_{F}\right) & =\frac{1}{L} \sum_{k<k_{F}} k C\left(\varepsilon_{k}\right) \\
& =\frac{1}{L} \sum_{k<0} k C\left(\varepsilon_{k}\right)+\frac{1}{L} \sum_{0<k<k_{F}} k \\
& =p(L)+\frac{2 \pi}{L^{2}} \sum_{l=1}^{n} l,
\end{aligned}
$$

where $n=\left\lfloor k_{F} L / 2 \pi\right\rfloor$. Using Eq. (B2), we then have

$$
p\left(L, k_{F}\right)=p(\infty)+\frac{2 \pi}{L^{2}}\left[\frac{1}{12}+\sum_{l=1}^{n} l\right]+O\left(\frac{1}{L^{4}}\right),
$$

where $p(\infty)$ is the momentum density at $L=\infty$ and $k_{F}=$ 0 . We see that the value of $h_{0}-c / 24$ is only equal to the idealized CFT result $h_{\sigma}-c / 24=1 / 12$ modulo 1 , while the integer part jumps periodically as a function of $k_{F}$ at fixed number of sites $L$, or as the number of sites $L$ at fixed $k_{F}$. Treating $k_{F}$ as fixed and valued in $(-\pi, \pi]$, the period in $L$ is given by $q=\left|2 \pi / k_{F}\right| \geqslant 2$, which need not be an integer. As described in Appendix A, the mod 1 ambiguity is attributed to $h_{0}$ rather than $c$, which corresponds to the topological spin $\theta_{0}=\theta_{\sigma}=e^{2 \pi i(1 / 8)}$.

The interpretation of Eq. (B4) is straightforward. As the number of sites $L$ increases, the single-particle momenta $(2 \pi / L) \mathbb{Z}_{L}$ become denser in the Brillouin zone $\mathbb{R} / 2 \pi \mathbb{Z}$. The $n$th jump in $h_{0}$ correspond to the motion of a single-particle state with momentum $2 \pi n / L$ through $k_{F}$ and into the Fermi sea, adding a momentum density $2 \pi n / L^{2}$ to the ground state.

Figure 8 presents the results of numerical computations of the momentum polarization Eq. (3) in a Chern insulator with $k_{F} \neq 0$ on a square lattice. Details of the model and 

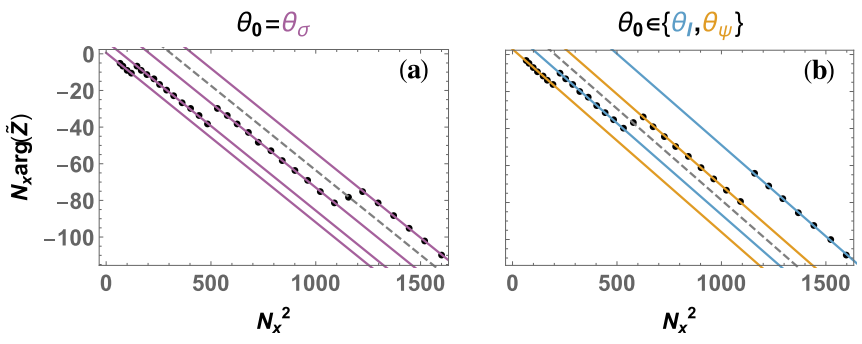

FIG. 8. Numerical results for the momentum polarization Eq. (3), in a Chern insulator with $k_{F} \neq 0$. Black dots mark numerically obtained values of $N_{x} \arg \tilde{Z}$ as a function of $N_{x}^{2}$. (a) Periodic boundary conditions. Purple lines indicate linear fits, with approximately the same slope and intercepts $2 \pi\left(1 / 12+\sum_{l=1}^{n} l\right)$ with the $y$ axis, with $n=0,1,2,3$, in accordance with Eq. (B4). This allows for the extraction of the topological spin $\theta_{\sigma}=e^{2 \pi i(1 / 8)}$. To illustrate the possibility of accidental degeneracies, we choose $k_{F}=3 / 34$, where a degeneracy occurs for $N_{x}=34$, and the average value of $N_{x} \arg \tilde{Z}$ between the two ground states is obtained. A gray dotted line indicates the average of the two neighboring purple lines. (b) Antiperiodic boundary conditions. Colored lines indicate linear fits, with approximately the same slope, and intercepts $2 \pi\left[-1 / 24+\sum_{l=1}^{n}(l-1 / 2)\right]$, with $n=1,2,3,4$, in accordance with Eq. (B5). The value $n=0$ is not obtained as it occurs only for small circumferences $N_{x}<6$ where $N_{x} \arg \tilde{Z}$ is not computed. Orange lines correspond to the fermion spin $\theta_{\psi}=-1$, while blue lines correspond to the vacuum spin $\theta_{I}=0$. To illustrate the possibility of accidental degeneracies, we choose $k_{F}=(5 / 2) / 24$, where an accidental degeneracy occurs for $N_{x}=24$.

computations can be found in the Supplemental Material [68]. In particular, Fig. 8(a) verifies Eq. (B4).

A subtle point, not mentioned above, is that when $k_{F} L / 2 \pi \in \mathbb{Z}$, which happens only when $k_{F} / 2 \pi=a / b$ is rational and $L \in b \mathbb{N}$, a single-particle state with momentum exactly $k_{F}$ exists, leading to an accidental degeneracy on the cylinder, between two many-body ground states with momentum densities given by Eq. (B4) with $n$ and $n+1$. The momentum polarization (3) then gives the average momentum density in the two ground states, as visualized by the gray dotted line in Fig. 8(a). For such system sizes the value $\theta_{0}=-\theta_{\sigma}$ may be obtained rather than the generic $\theta_{0}=\theta_{\sigma}$.

The same analysis can be performed for antiperiodic boundary conditions, where we sum over single-particle momenta $k \in \frac{2 \pi}{L}\left(\mathbb{Z}_{L}+\frac{1}{2}\right)$. Equation (B4) is then modified to

$$
p\left(L, k_{F}\right)=p(\infty)+\frac{2 \pi}{L^{2}}\left[-\frac{1}{24}+\sum_{l=1}^{n}\left(l-\frac{1}{2}\right)\right]+O\left(\frac{1}{L^{4}}\right),
$$

where $n=\left\lfloor\frac{k_{F} L}{2 \pi}-\frac{1}{2}\right\rfloor$. As a function of $L$, jumps in $h_{0}-$ $c / 24$ occur with the same period $q=\left|2 \pi / k_{F}\right| \geqslant 2$, but are shifted by $q / 2$. Moreover, $h_{0}-c / 24$ now attains two values modulo 1 , namely, $h_{I}-c / 24=-1 / 24$ and $h_{\psi}-c / 24=$ $1 / 2-1 / 24$.

Equation (B5) therefore demonstrates explicitly the statements made in Appendix A 1. For $k_{F}=0$, the cylinder ground state of the Chern insulator corresponds to the idealized Weyl fermion CFT. A single value $h_{0}=0$ is attained, which is the conformal weight $h_{I}$ of the CFT vacuum. A nonvanishing $k_{F}$ corresponds to the addition of a chemical potential to the CFT, which changes the energies of the CFT states, favoring a CFT exited state over the CFT vacuum. The cylinder ground state of the Chern insulator may then correspond to any CFT state in the conformal family of either the vacuum $I$ or fermion $\psi$, which need not be primary. From the bulk TFT perspective, we see that $\theta_{0}=e^{2 \pi i h_{0}}$ may be equal to either of the topological spins $\theta_{I}=1, \theta_{\psi}=-1$ as a function of $L$.

As in the case of periodic boundary conditions, accidental degeneracies on the cylinder occur when $k_{F} L / 2 \pi \in \mathbb{Z}+1 / 2$, changing the value of $h_{0}$ attained from the momentum polarization to its average over the degenerate states. For such system sizes, the value $\theta_{0}= \pm \sqrt{\theta_{I} \theta_{\psi}}$ is obtained than the generic $\theta_{0} \in\left\{\theta_{I}, \theta_{\psi}\right\}$.

Equation (B5) is verified numerically in Fig. 8(b), which demonstrates that the value of $\theta_{0}=e^{2 \pi i h_{0}}$, obtained from the momentum polarization (3), takes different values in the set $\left\{\theta_{a}\right\}$ of topological spins as a function of system size $L$, apart from accidental degeneracies.

\section{No finite-size correction at finite temperature}

The line $y=0$ where $T_{R}$ jumps can be interpreted as an additional boundary component at the "entanglement temperature" $\beta_{*}^{-1}$. Reference [40] used the modular transformations of CFT partition functions to demonstrate that when $\beta_{*} \ll$ $L / v$, this boundary component does not contribute to the $1 / L$ correction to $\log \tilde{Z}$. Here we note that the same result holds for free fermions with a general dispersion $\varepsilon_{k}$. The contribution of the additional boundary component to $\log \tilde{Z}$ is given by $\log \tilde{Z}_{*}(L)=L f_{*}(L)$, with the free energy density

$$
f_{*}(L)=\frac{1}{L} \sum_{k} \log \left(1+e^{i a k} e^{-\beta_{*} \varepsilon_{k}}\right) C\left(\varepsilon_{k}\right) .
$$

Using Eq. (B1) one finds $f_{*}(L)=f_{*}(\infty)+O\left(L^{-4}\right)$ for both periodic and antiperiodic boundary conditions, which implies $\log \tilde{Z}_{*}=L f_{*}(\infty)+O\left(L^{-3}\right)$, with no $1 / L$ contribution. The complex number $f_{*}(\infty)=\int \log \left(1+e^{i a k} e^{-\beta_{*} \varepsilon_{k}}\right) C\left(\varepsilon_{k}\right) d k / 2 \pi$ contributes to the nonuniversal $\alpha$ in Eq. (3).

\section{APPENDIX C: CUTTING THE TORUS ALONG AN ARBITRARY VECTOR}

For $\mathbf{d}=\left(d_{x}, 0\right)$ we restrict to $N_{x}=n_{x} d_{x}, n \in \mathbb{N}$, viewing $d_{x}$ as an enlarged lattice spacing, and treating $n$ as a reduced number of sites along the circumference in place of $N_{x}$. The same logic applies to $\mathbf{d}=\left(0, d_{y}\right)$. For $\mathbf{d}=\left(d_{x}, d_{y}\right)$ with both $d_{x}, d_{y} \neq 0$, we restrict to system sizes $\left(N_{x}, N_{y}\right)=n \mathbf{d}$, such that the line $l=\operatorname{span}_{\mathbb{R}} \mathbf{d}$ is a diagonal of the rectangle $n N_{x} \times n N_{y}$ and corresponds to a circle on the torus $\left(\mathbb{R} / N_{x} \mathbb{Z}\right) \times\left(\mathbb{R} / N_{y} \mathbb{Z}\right)$. Cutting $X$ along this line produces a cylinder $C$ of circumference $L=n|\mathbf{d}|$. We then view $|\mathbf{d}|$ as a lattice spacing and $n$ as $a$ the number of sites along the circumference. Note that the distance between the boundary components of the resulting cylinder is $R=n d_{x} d_{x} /|\mathbf{d}|$, and the thermodynamic limit is indeed obtained as $n \rightarrow \infty$. With these identifications the momentum polarization (3) remains unchanged, apart from a modification of the nonuniversal $\alpha$ to $\alpha|\mathbf{d}|^{2}$. 


\section{APPENDIX D: DEALING WITH ACCIDENTAL DEGENERACIES ON THE CYLINDER}

As demonstrated in Appendix B, for certain system sizes $N_{x}$ accidental degeneracies occur on the cylinder, and the function $\theta_{0}\left(N_{x}\right)=e^{2 \pi i h_{0}\left(N_{x}\right)}$ obtained from Eq. (3) may take values outside the set $\left\{\theta_{a}\right\}$, namely, $\theta_{0}= \pm \sqrt{\theta_{a} \theta_{b}}$ for a twofold degeneracy. In this Appendix we complete the derivation of Results 1 and $1 \mathrm{~F}$ by considering the possibility of such degeneracies.

First, even in the presence of degeneracies, $\theta_{0}$ is valued in a finite set. Therefore, Eq. (6) still implies that $\epsilon^{\prime}=n / m$ is rational, and that $\theta_{0}\left(N_{x}\right) e^{-2 \pi i c / 24}=e^{-2 \pi i \epsilon^{\prime} N_{x}^{2}}$ periodically covers a subset $S \ni 1$ of $m$ th roots of unity, for all large enough $N_{x}$. We denote by $\mathcal{N} \subset \mathbb{N}$ the set of circumferences $N_{x}$ for which a degeneracy appears and $\theta_{0}\left(N_{x}\right) \notin\left\{\theta_{a}\right\}$. If circumferences $N_{x} \in m \mathbb{N}$, where $e^{2 \pi i \epsilon^{\prime} N_{x}^{2}}=1$, are not all contained in $\mathcal{N}$, then $1=\theta_{0}\left(N_{x}\right) e^{-2 \pi i c / 24} \in\left\{\theta_{a} e^{-2 \pi i c / 24}\right\}$, as stated in Results 1 and $1 \mathrm{~F}$.

We are left with the complementary case, where degeneracies occur for all $N_{x} \in m \mathbb{N}$, i.e., $m \mathbb{N} \subset \mathcal{N}$. Note that this case is highly fine tuned, as it ties together the nonuniversal $\epsilon^{\prime}=n / m$ and the set $\mathcal{N}$ of $N_{x}$ s where accidental degeneracies appear. In order to deal with this case, we make use of the Frobenius-Perron theorem to resolve the degenerate ground-state subspace, without introducing signs. The analysis applies only to the bosonic setting of Result 1 and is similar to that made in Sec. VII. We now have a Hamiltonian $H^{\prime}$ on the cylinder, which has an exactly degenerate ground-state subspace for all $N_{x} \in m \mathbb{N}$ and has nonpositive matrix elements in the on-site homogenous basis $|s\rangle$. The Frobenius-Perron theorem implies that an orthonormal basis $|i\rangle$ with non-negative entries may be chosen for the ground-state subspace, $\langle s \mid i\rangle \geqslant 0$ for all $s, i$. It follows that the matrix elements of $T_{R}$ in the basis $|i\rangle$ are non-negative, $M_{i j}:=\left\langle i\left|T_{R}\right| j\right\rangle \geqslant 0$. Taking the $N_{x}$ th matrix power of $M$ we have $\left(M^{N_{x}}\right)_{i j} \geqslant 0$. Equation (3) implies that the eigenvalues of $M^{N_{x}}$ are of the form $e^{-\delta^{\prime} N_{x}^{2}} e^{-2 \pi i \epsilon^{\prime} N_{x}^{2}+o(1)} \theta_{a} e^{-2 \pi i c / 24}$, so we can write $\left(M^{N_{x}}\right)_{i j}=e^{-\delta^{\prime} N_{x}^{2}} e^{-2 \pi i \epsilon^{\prime} N_{x}^{2}+o(1)} T_{i j}$, where $T_{i j}$ has eigenvalues $\{\lambda\} \subset\left\{\theta_{a} e^{-2 \pi i c / 24}\right\}$. In particular, $T_{i j}$ is unitary. Since $e^{2 \pi i \epsilon^{\prime} N_{x}^{2}}=1$ for all $N_{x} \in m \mathbb{N}$, we see that $T_{i j}$ also has non-negative entries and is therefore a permutation matrix, containing 1 in its spectrum (see Sec. VII). It follows that $1 \in\{\lambda\} \subset\left\{\theta_{a} e^{-2 \pi i c / 24}\right\}$, asserting Result 1 .

We are currently unaware of an analog of the FrobeniusPerron theorem in the context of DQMC, that may be used to resolve the degenerate ground-state subspace without introducing signs. Instead, we will make a physical assumption under which Result 1F holds. Namely, we will assume that the fine-tuned constraint $\epsilon^{\prime}=n / m$ and $m \mathbb{N} \subset \mathcal{N}$ may be lifted by a sign-free perturbation. This includes (i) perturbations to the effective single-fermion Hamiltonian $h_{\phi(\tau)}$ that do not violate the algebraic condition $h_{\phi(\tau)} \in \mathcal{C}_{h}$, (ii) perturbations to the bosonic action $S_{\phi}$ that maintain its reality, and (iii) changes of the vector $\mathbf{d}$ along which the torus is cut to a cylinder, as described in Appendix $\mathrm{C}$, which will generically change the details of the boundary spectrum, including the nonuniversal number $\epsilon^{\prime}$ and the set $\mathcal{N}$ of $N_{x}$ 's where accidental degeneracies appear. A robustness of $\epsilon^{\prime}$ and the set $\mathcal{N}$, both nonuniversal, under all three of the above deformations certainly goes beyond the low-energy description of a chiral TFT in the bulk and a chiral CFT on the boundary. We also adopt this assumption in the fermionic spontaneously chiral setting of Result $2 \mathrm{~F}$.

A stoquastic variant of the above assumption may also be adopted to establish the bosonic spontaneously chiral Result 2 , but a stronger statement can in fact be made, by again making use of the Frobenius-Perron theorem to resolve the accidentally degenerate ground states. The Frobenius-Perron theorem does not immediately complete the derivation of Result 2, since the former is a ground-state statement, while the latter made use of the finite temperature $\Delta E \ll \beta^{-1} \ll$ $N_{x}^{-1}$, where $\Delta E$ is the exponentially small finite-size splitting between low-lying symmetry-breaking eigenstates. This difficulty does not arise in the "classical symmetry-breaking" scenario, where $\Delta E=0$. In the generic case $\Delta E \neq 0$, we can make progress under the assumption that the $\mathcal{T}, \mathcal{P}$-even state $W[|+\rangle+|-\rangle]$ has lower energy than the $\mathcal{T}, \mathcal{P}$-odd state $W[|+\rangle-|-\rangle]$, rather than the opposite possibility. The derivation of Result 2 in Sec. IV A can then be repeated at zero temperature. In particular, Eq. (10) and its analysis are unchanged.

\section{APPENDIX E: A "NONLOCAL DESIGN PRINCIPLE" FOR CHIRAL TOPOLOGICAL MATTER}

As stated in Sec. V C, the composition $T=\mathcal{P}^{(0)} \mathcal{T}^{(1 / 2)}$ of the spin-less reflection with the spin-1/2 time reversal naturally provides a design principle for a class of models for chiral topological matter. Here we describe such T-invariant models for chiral topological superconductors.

The simplest model comprises two copies, labeled by $\sigma=\uparrow, \downarrow$, of a spin-less $p+i p$ superconductor,

$$
H=\sum_{\mathbf{x}, \mathbf{x}^{\prime}, \sigma, \sigma^{\prime}}\left[\psi_{\sigma, \mathbf{x}}^{\dagger} h_{\mathbf{x}, \mathbf{x}^{\prime}, \sigma, \sigma^{\prime}} \psi_{\sigma, \mathbf{x}^{\prime}}+\psi_{\sigma, \mathbf{x}}^{\dagger} \Delta_{\mathbf{x} \mathbf{x}^{\prime}, \sigma, \sigma^{\prime}} \psi_{\sigma, \mathbf{x}^{\prime}}^{\dagger}+\text { H.c. }\right] \text {. }
$$

Here

$$
\Delta=\left(\begin{array}{cc}
\Delta_{0}\left(d^{x}+i d^{y}\right) & 0 \\
0 & \Delta_{0}\left(d^{x}+i d^{y}\right)
\end{array}\right),
$$

where $d_{\mathbf{x x}^{\prime}}^{x}\left(d_{\mathbf{x x}^{\prime}}^{y}\right)$ is the antisymmetric $x(y)$ difference operator,

$$
\begin{aligned}
& d_{\mathbf{x x}^{\prime}}^{x}=\left(\delta_{x, x^{\prime}+1}-\delta_{x+1, x}\right) \delta_{y, y^{\prime}} / 2, \\
& d_{\mathbf{x x}^{\prime}}^{y}=\delta_{x, x^{\prime}}\left(\delta_{y, y^{\prime}+1}-\delta_{y+1, y^{\prime}}\right) / 2,
\end{aligned}
$$

and $\Delta_{0} \in \mathbb{R}-\{0\}$. Additionally,

$$
h=\left(\begin{array}{ll}
t & 0 \\
0 & t
\end{array}\right),
$$

and the hopping $t$ is real and reflection symmetric, e.g.,

$$
t_{\mathbf{x}, \mathbf{x}^{\prime}}=\frac{1}{2} t_{0}\left(\delta_{x, x^{\prime}+1}+\delta_{x+1, x^{\prime}}\right) \delta_{y, y^{\prime}}+(x \leftrightarrow y)-\mu,
$$

with $t_{0}>0, \mu \in \mathbb{R}$. It is well known that the chemical potential $\mu$ can be used to tune the model between gapped SPT phases with $c=0,-1,1$, for $|\mu|>2 t_{0},-2 t_{0}<\mu<$ $0,0<\mu<2 t_{0}$, respectively; see, e.g., Ref. [93]. Additionally, the Hamiltonian is invariant under the combination of the unitary spin-less reflection $\mathcal{P}^{(0)}: \psi_{\sigma,(x, y)} \mapsto \psi_{\sigma,(x,-y)}$ and the antiunitary spin-full time reversal $\mathcal{T}^{(1 / 2)}: \psi_{\uparrow, \mathbf{x}} \mapsto \psi_{\downarrow, \mathbf{x}}$, $\psi_{\downarrow, \mathbf{x}} \mapsto-\psi_{\uparrow, \mathbf{x}} \cdot$ 
The model can be written in the BdG form

$$
H=\sum_{\mathbf{x}, \mathbf{x}^{\prime}} \Psi_{\mathbf{x}}^{\dagger} h_{\mathrm{BdG}}^{\mathbf{x}, \mathbf{x}^{\prime}} \Psi_{\mathbf{x}^{\prime}}
$$

where $\Psi_{\mathbf{x}}^{T}=\left(\psi_{\uparrow \mathbf{x}}, \psi_{\downarrow \mathbf{x}}, \psi_{\uparrow \mathbf{x}}^{\dagger}, \psi_{\downarrow \mathbf{x}}^{\dagger}\right)$ is the Nambu spinor (a Majorana spinor), and

$$
h_{\mathrm{BdG}}=\left(\begin{array}{cc}
h & \Delta \\
-\Delta^{*} & -h^{*}
\end{array}\right) .
$$

The "single-fermion" space on which $h_{\mathrm{BdG}}$ acts is $\mathcal{H}_{1 \mathrm{~F}}=$ $\mathcal{H}_{X} \otimes \mathcal{H}_{\text {spin }} \otimes \mathcal{H}_{\text {Nambu }} \cong \mathbb{C}^{|X|} \otimes \mathbb{C}^{2} \otimes \mathbb{C}^{2}$. The spin-less re- flection acts on $\mathcal{H}_{1 \mathrm{~F}}$ as $\mathcal{P}^{(0)}=\mathcal{P}_{X}^{(0)} \otimes I_{2} \otimes I_{2}$, where $\mathcal{P}_{X}^{(0)}=$ $\delta_{x, x^{\prime}} \delta_{y,-y^{\prime}}$. The spin-full time-reversal acts by $\mathcal{T}^{(1 / 2)}=I_{|X|} \otimes$ $i Y \otimes I_{2} \mathcal{K}$, where $Y$ is the Pauli matrix and $\mathcal{K}$ is the complex conjugation. The operator $T=\mathcal{P}^{(0)} \mathcal{T}^{(1 / 2)}$ satisfies $\mathrm{T}^{2}=-I$ and $\left[\mathrm{T}, h_{\mathrm{BdG}}\right]=0$ and is therefore a time-reversal design principle which applies to $h_{\mathrm{BdG}}$, implying $\operatorname{det}\left(\partial_{\tau}+h_{\mathrm{BdG}}\right) \geqslant$ 0 . Since $h_{\mathrm{BdG}}$ acts on the Majorana spinor $\Psi$, the relevant quantity is actually the Pfaffian $\operatorname{Pf}\left(\partial_{\tau}+h_{\mathrm{BdG}}\right)=$ $\sqrt{\operatorname{det}\left(\partial_{\tau}+h_{\mathrm{BdG}}\right)} \geqslant 0$, where the principal branch of the square root is chosen.

The Hamiltonian $h_{\mathrm{BdG}}$ can be considerably generalized while maintaining $\left[\mathrm{T}, h_{\mathrm{BdG}}\right]=0$, by taking

$$
h=\left(\begin{array}{cc}
t & r \\
-r^{*} & t^{*}
\end{array}\right), \quad \Delta=\left(\begin{array}{cc}
e^{i \alpha}\left(\left|\Delta_{x}\right| d^{x}+i\left|\Delta_{y}\right| d^{y}\right)^{|\ell|} & e^{i \tilde{\alpha}}\left(\left|\tilde{\Delta}_{x}\right| d^{x}+i\left|\tilde{\Delta}_{y}\right| d^{y}\right)^{|\tilde{\ell}|} \\
-e^{-i \tilde{\alpha}}\left(\left|\tilde{\Delta}_{x}\right| d^{x}+i\left|\tilde{\Delta}_{y}\right| d^{y}\right)^{|\tilde{\ell}|} & e^{-i \alpha}\left(\left|\Delta_{x}\right| d^{x}+i\left|\Delta_{y}\right| d^{y}\right)^{|\ell|}
\end{array}\right),
$$

where $t_{\mathbf{x}, \mathbf{x}^{\prime}}, r_{\mathbf{x}, \mathbf{x}^{\prime}}$ are general matrices, and $\ell \in 2 \mathbb{Z}+1(\tilde{\ell} \in$ $2 \mathbb{Z}$ ) is the angular momentum channel of the triplet (singlet) pairing. This can be further generalized to a sum over all angular momentum channels $\sum_{\ell \in 2 \mathbb{Z}+1} e^{i \alpha_{\ell}}\left(\left|\Delta_{\ell, x}\right| d^{x}+i\left|\Delta_{\ell, y}\right| d^{y}\right)^{|\ell|}$ and similarly for $\tilde{\ell}$. The model is Hermitian for $t=t^{\dagger}, r=$ $-r^{T}$, but this is not required to avoid the sign problem.

In order to obtain an interacting model, the parameters $\phi=\left\{t, r, \alpha_{\ell}, \tilde{\alpha}_{\tilde{\ell}},\left|\Delta_{\ell, x}\right|,\left|\Delta_{\ell, y}\right|,\left|\tilde{\Delta}_{\tilde{\ell}, x}\right|,\left|\tilde{\Delta}_{\tilde{\ell}, y}\right|\right\}$ can now be promoted to space-time-dependent bosonic fields, with any action $S_{\phi} \in \mathbb{R}$. The model will be sign-free as long as $h_{\mathrm{BdG}}$ remains T-invariant for all configurations $\phi$, which requires that only reflection-even configurations $\phi(\tau, x, y)=\phi(\tau, x,-y)$ are summed over. As discussed in Sec. VC, this implies nonlocal interactions, which effectively fold the chiral system into a nonchiral system on half of space.

\section{APPENDIX F: LOCALITY AND HOMOGENEITY OF KNOWN DESIGN PRINCIPLES}

In this Appendix we review all fermionic design principles known to us, clarify their common features, and describe the conditions under which they are on-site homogeneous, imply a termwise sign-free DQMC representation, and allow a locally sign-free DQMC simulation, as defined in Sec. VB. The design principles are stated as algebraic conditions satisfied by the effective single-fermion Hamiltonian $h_{\phi}=h_{\phi(\tau)}$ and the corresponding imaginary-time evolution $U_{\phi}=\mathrm{TO} e^{-\int_{0}^{\beta} h_{\phi(\tau)} \mathrm{d} \tau}$, or in terms of the operator $D_{\phi}=\partial_{\tau}+h_{\phi}$; see Sec. V A.

Contraction semigroups and Majorana time reversals. The time-reversal design principle covered in Sec. V C is a special case of a broad class of design principles that were recently discovered and unified [22-24]. These are stated in terms of Majorana fermions, where $\psi$ is real and $\bar{\psi}=\psi^{T}$, in which case $h_{\phi}$ is antisymmetric and the determinants in (17) are replaced by their square roots. Reference [24] shows that if

$$
\begin{gathered}
\mathrm{J}_{1} h_{\phi}-h_{\phi}^{*} \mathrm{~J}_{1}=0, \\
i\left(\mathrm{~J}_{2} h_{\phi}-h_{\phi}^{*} \mathrm{~J}_{2}\right) \geqslant 0,
\end{gathered}
$$

where the matrices $J_{1}, J_{2}$ are real and orthogonal, and obey $J_{1}^{T}= \pm J_{1}, J_{2}^{T}=-J_{2},\left\{J_{1}, J_{2}\right\}=0$, then $\operatorname{Det}\left(I+U_{\phi}\right) \geqslant 0$.
The equality (F1) corresponds to an antiunitary symmetry $\mathrm{T}_{1}=\mathrm{J}_{1} \mathcal{K}, \mathrm{T}_{1}^{2}= \pm I$, where $\mathcal{K}$ is the complex conjugation. If the inequality (F2) is replaced by an equality, it corresponds to an additional antiunitary symmetry, $\mathrm{T}_{2}=\mathrm{J}_{2} \mathcal{K}, \mathrm{T}_{2}^{2}=-I$. The case $T_{1}^{2}=-I$ then reduces to the standard time-reversal $\mathrm{T}$ described in Sec. VC, while $\mathrm{T}_{1}^{2}=I$ corresponds to the "Majorana class" of Ref. [22]. More generally, the inequality (F2) states that the left-hand side is a positive semidefinite matrix and implies that $h_{\phi}$ is a generator of the contraction semigroup defined by the Hermitian metric $\eta_{2}=i J_{2}, \eta_{2}^{2}=I$, $\left[\mathrm{T}_{1}, \eta_{2}\right]=0$. Explicitly, Eqs. (F1) and (F2) can be written as

$$
\left[\mathrm{T}_{1}, h_{\phi}\right]=0, \quad \eta_{2} h_{\phi}+h_{\phi}^{\dagger} \eta_{2} \geqslant 0,
$$

and imply

$$
\left[\mathrm{T}_{1}, U_{\phi}\right]=0, \quad \eta_{2}-U_{\phi}^{\dagger} \eta_{2} U_{\phi} \geqslant 0 .
$$

In the language of Sec. VB, for fixed $\mathrm{T}_{1}, \eta_{2}$, the set $\mathcal{C}_{h}$ contains all matrices $h_{\phi}$ satisfying (F3). It is clear that this set is additive: $h_{1}+h_{2} \in \mathcal{C}_{h}$ for all $h_{1}, h_{2} \in \mathcal{C}_{h}$. The set $\mathcal{C}_{U}$ contains all matrices $U_{\phi}$ satisfying Eq. (F4), and is multiplicative: $U_{1} U_{2} \in \mathcal{C}_{U}$ for all $U_{1}, U_{2} \in \mathcal{C}_{U}$

A sufficient condition on $T_{1}, \eta_{2}$ that guarantees that the design principle they define is on-site homogenous is that they are of the form $\mathrm{T}_{1}=I_{|X|} \otimes \mathrm{t}_{1}, \eta_{2}=I_{|X|} \otimes e_{2}$, written in terms of the decomposition $\mathcal{H}_{1 \mathrm{~F}} \cong \mathbb{C}^{|X|} \otimes \mathbb{C}^{\mathrm{d}_{\mathrm{F}}}$ of the single-fermion space. The permutation matrices $O^{(\sigma)}$ defined in Eq. (20) then commute with $\eta_{2}$ and $\mathrm{T}_{1}$. Since $O^{(\sigma)}$ are also unitary, we have $O^{(\sigma)} \in \mathcal{C}_{U}$ for all $\sigma \in S_{X}$. All examples described in Refs. [22-24] are of the on-site homogenous form $T_{1}=$ $I_{|X|} \otimes \mathrm{t}_{1}, \eta_{2}=I_{|X|} \otimes e_{2}$.

As in our discussion of $\mathrm{T}$ in Sec. VC, the locality of $\mathrm{T}_{1}=$ $I_{|X|} \otimes \mathrm{t}_{1}$ means that it can be applied termwise, by symmetrizing the local terms $h_{\phi ; \mathbf{x}} \mapsto \frac{1}{2}\left(h_{\phi ; \mathbf{x}}+\mathrm{T}_{1} h_{\phi ; \mathbf{x}} \mathrm{T}_{1}^{-1}\right)$. A similar procedure for $\eta_{2}$ is possible only if the inequality in Eq. (F3) holds as an equality (as in Ref. [22]). The contraction semigroup defined by $\eta_{2}$ then reduces to an orthogonal group, and one can enforce the termwise relations $\eta_{2} h_{\phi ; \mathbf{x}}+h_{\phi ; \mathbf{x}}^{\dagger} \eta_{2}=0$ by $h_{\phi ; \mathbf{x}} \mapsto \frac{1}{2}\left(h_{\phi ; x}-\eta_{2} h_{\phi ; x}^{\dagger} \eta_{2}\right)$.

Collecting the above, we see that if $\mathrm{T}_{1}, \eta_{2}$ can be brought to the form $T_{1}=I_{|X|} \otimes t_{1}, \eta_{2}=I_{|X|} \otimes e_{2}$ by the same 
single-fermion local unitary $u$, then a DQMC representation which is $T_{1}$-symmetric, and respects Eq. (F3) termswise, leads to a locally sign-free DQMC simulation.

Split orthogonal group. Another recently discovered design principle is defined in terms of the split orthogonal group $O(n, n)$ [18]: if $U_{\phi} \in O(n, n)$, then the sign of $\operatorname{Det}\left(I+U_{\phi}\right)$ depends only on the connected component of $O(n, n)$ to which $U_{\phi}$ belongs. If the sign of $e^{-S_{\phi}}$ is manifestly compatible with the connected component of $U_{\phi}$ in $O(n, n)$, one has $p(\phi)=$ $e^{-S_{\phi}} \operatorname{Det}\left(I+U_{\phi}\right) \geqslant 0$. More explicitly, the statement $U_{\phi} \in$ $O(n, n)$ implies that $U_{\phi}$ is a real matrix and $\eta-U_{\phi}^{T} \eta U_{\phi}=0$, where $\eta=\operatorname{diag}\left(I_{n},-I_{n}\right)$. Restricting to the identity component $O_{0}(n, n)$, this amounts to the statements that $h_{\phi}$ is in the Lie algebra $o(n, n)$ : it is real and satisfies $\eta h_{\phi}+h_{\phi}^{T} \eta=0$.

In a basis-independent formulation, the data that define the design principle are an antiunitary $\tilde{\mathrm{T}}$, such that $\tilde{\mathrm{T}}^{2}=I$, and a Hermitian metric $\tilde{\eta}$ with canonical form $\eta$, such that $[\tilde{T}, \tilde{\eta}]=$ 0 . The set $\mathcal{C}_{h}$ is then given by matrices $h_{\phi}$ satisfying

$$
\left[\tilde{\mathrm{T}}, h_{\phi}\right]=0, \quad \tilde{\eta} h_{\phi}+h_{\phi}^{\dagger} \tilde{\eta}=0,
$$

while $\mathcal{C}_{U}$ is defined by

$$
\left[\tilde{\mathrm{T}}, U_{\phi}\right]=0, \quad \tilde{\eta}-U_{\phi}^{\dagger} \tilde{\eta} U_{\phi}=0 .
$$

The analogy with (F3) and (F4) is now manifest, with the inequalities strengthened to equalities. Accordingly, the $O(n, n)$ design principle is on-site homogeneous if $\tilde{\mathrm{T}}=I_{|X|} \otimes \tilde{\mathrm{t}}$ and $\tilde{\eta}=I_{|X|} \otimes \tilde{e}$. If these forms can be obtained by conjugation of $\tilde{\mathrm{T}}, \tilde{\eta}$ with the same single-fermion local unitary $u$, then a DQMC representation which is sign-free due to $\tilde{\mathrm{T}}, \tilde{\eta}$ leads to a locally sign-free DQMC simulation.

The above statements hold for $U_{\phi}$ in the identity component $O_{0}(n, n)$, which is always the case when $h_{\phi} \in o(n, n)$ and $U_{\phi}=\mathrm{TO} e^{-\int_{0}^{\beta} h_{\phi(\tau)} \mathrm{d} \tau}$. Time evolutions in the additional three connected components of $O(n, n)$ can be obtained by operator insertions generalizing $U_{\phi}=U_{k} \cdots U_{2} U_{1}$ to $U_{k} \cdots O_{2} U_{2} O_{1} U_{1}$, where $O \in O(n, n) / O_{0}(n, n)$ [18]. These can be incorporated into the framework of Sec. V, if each $O_{k}$ is supported on a disk of radius $w$ around a site $\mathbf{x}_{k}$, i.e., $\left(O_{k}\right)_{\mathbf{x}, \mathbf{y}}=\delta_{\mathbf{x}, \mathbf{y}}$ if $\left|\mathbf{x}-\mathbf{x}_{k}\right|>w$ or $\left|\mathbf{y}-\mathbf{x}_{k}\right|>w$. With this generalization, all sign-free examples described in Ref. [18] amount to locally sign-free DQMC.

Solvable fermionic and bosonic actions. Reference [21] described a design principle that nontrivially relates the fermionic action $S_{\psi, \phi}=\bar{\psi} D_{\phi} \psi$ and bosonic action $S_{\phi}$. A fermionic action was termed "solvable" if $D_{\phi}$ has the form

$$
D_{\phi}=\left(\begin{array}{cc}
0 & M_{\phi} \\
-M_{\phi}^{\dagger} & 0
\end{array}\right),
$$

which clearly implies $\operatorname{Det}\left(D_{\phi}\right)=\left|\operatorname{Det}\left(M_{\phi}\right)\right|^{2} \geqslant 0$. Here the imaginary time circle $\mathbb{R} / \beta \mathbb{Z}$ is discretized to $\mathbb{Z}_{\beta}=\mathbb{Z} / \beta \mathbb{Z}$, and $D_{\phi}$ is treated as a matrix on $\mathbb{C}^{\beta} \times \mathcal{H}_{1 \mathrm{~F}}=\mathbb{C}^{\beta} \times \mathbb{C}^{|X|} \times$
$\mathbb{C}^{\mathrm{d}_{F}}$, with indices $(\tau, \mathbf{x}, \alpha),\left(\tau^{\prime}, \mathbf{x}^{\prime}, \alpha^{\prime}\right)$ for time, space, and internal degrees of freedom. For example, the Hamiltonian form $D_{\phi}=\partial_{\tau}+h_{\phi(\tau)}$ is discretized to

$$
\begin{aligned}
& {\left[D_{\phi}\right]_{(\tau, \mathbf{x}, \alpha),\left(\tau^{\prime}, \mathbf{x}^{\prime}, \alpha^{\prime}\right)}} \\
& \quad=\left(\delta_{\tau, \tau^{\prime}}-\delta_{\tau-1, \tau^{\prime}}\right) \delta_{\mathbf{x}, \mathbf{x}^{\prime}} \delta_{\alpha, \alpha^{\prime}}+\delta_{\tau-1, \tau^{\prime}}\left[h_{\phi(\tau)}\right]_{(\mathbf{x}, \alpha),\left(\mathbf{x}^{\prime}, \alpha^{\prime}\right)} .
\end{aligned}
$$

In a basis-independent language, Eq. (F7) corresponds to

$$
\left\{\Gamma, D_{\phi}\right\}=0, \quad D_{\phi}^{\dagger}=-D_{\phi},
$$

where $\Gamma$ is a "chiral symmetry", $\Gamma^{2}=I, \Gamma=\Gamma^{\dagger}$. Equation (19) is then obtained in a basis where $\Gamma=\operatorname{diag}(I,-I)$. Note, however, that $\Gamma$ acts on $D_{\phi}$ rather than $h_{\phi}$, and that the form (F7) requires a noncanonical transformation away from the Hamiltonian form $(\mathrm{F} 8)$. We refer to $\Gamma$ as onsite homogeneous if it is of the form $\Gamma=I_{\beta} \otimes I_{|X|} \otimes \gamma$, and to $D_{\phi}$ as local if $D_{\phi}=\sum_{\tau, \mathbf{x}} D_{\phi ; \tau, \mathbf{x}}$ where each term $D_{\phi ; \tau, \mathbf{x}}$ is supported on a disk of radius $r$ around $(\tau, \mathbf{x})$ and depends on the values of $\phi$ at points within this disk. The action $D_{\phi}$ is "termwise solvable" if each $D_{\phi ; \tau, \mathbf{x}}$ satisfies (F9). Any local $D_{\phi}$ obeying (F9) with $\Gamma=I_{\beta} \otimes I_{|X|} \otimes$ $\gamma$ can be made termwise solvable by replacing $D_{\phi ; \tau, \mathbf{x}} \mapsto$ $\frac{1}{2}\left(D_{\phi ; \tau, \mathbf{x}}-\Gamma D_{\phi ; \tau, \mathbf{x}} \Gamma\right)$ and then $D_{\phi ; \tau, \mathbf{x}} \mapsto \frac{1}{2}\left(D_{\phi ; \tau, \mathbf{x}}-D_{\phi ; \tau, \mathbf{x}}^{\dagger}\right)$. The twisted fermionic boundary conditions in (22) are implemented by declaring that the index " $(\tau=0, \mathbf{x}, \alpha)$ " that appears in Eq. (F8) corresponds to $(\tau=\beta, x+\lambda \Theta(y), y, \alpha)$ with $\lambda \neq 0$. Equation (F9) then holds for all $\lambda$ if $\Gamma=I_{\beta} \otimes$ $I_{|X|} \otimes \gamma$. Under these conditions, solvable fermionic actions can then be incorporated into the definition of locally sign-free DQMC given in Sec. V.

All examples given in Ref. [21] have an on-site $\Gamma$ and local $D_{\phi}$, and it follows from the above discussion that, under these conditions, solvable fermionic actions can then be incorporated into the definition of locally sign-free DQMC given in Sec. V.

A bosonic action $S_{\phi}$ for a complex valued field $\phi=|\phi| e^{i \theta}$ was termed "solvable" in Ref. [21] if

$$
S_{\phi}=S_{|\phi|}-\sum_{u, u^{\prime}} \beta_{u, u^{\prime}}\left|\phi_{u}\right|\left|\phi_{u^{\prime}}\right| \cos \left(\varepsilon_{u} \theta_{u}+\varepsilon_{u^{\prime}} \theta_{u^{\prime}}\right),
$$

where $u=(\mathbf{x}, \tau), u=\left(\mathbf{x}^{\prime}, \tau^{\prime}\right)$, and $\varepsilon_{u}, \varepsilon_{u^{\prime}} \in\{ \pm 1\}$, and $\beta_{u, u^{\prime}} \geqslant$ 0 . For such actions, it was shown that all correlators $\int D \phi e^{-S_{\phi}} \phi_{u_{1}} \cdots \phi_{u_{k}}$ are non-negative, and therefore $\phi$ can be added to the diagonal in (F7) with a positive coupling constant

$$
D_{\phi}=\left(\begin{array}{cc}
g \phi & M \\
-M^{\dagger} & g \phi
\end{array}\right), \quad g>0
$$

without introducing signs, though $D_{\phi}$ is no longer solvable. Solvable bosonic actions are easily incorporated into the framework of Sec. V, as long as they are local in the sense of Eq. (19), and in particular, $\beta_{u, u^{\prime}}=0$ unless the points $u$ and $u^{\prime}$ are close.
[1] N. Metropolis and S. Ulam, The Monte Carlo method, J. Am. Stat. Assoc. 44, 335 (1949).

[2] M. Troyer and U.-J. Wiese, Computational Complexity and Fundamental Limitations to Fermionic Quantum Monte Carlo Simulations, Phys. Rev. Lett. 94, 170201 (2005).
[3] F. Barahona, On the computational complexity of Ising spin glass models, J. Phys. A: Math. Gen. 15, 3241 (1982).

[4] F. F. Assaad and H. G. Evertz, World-line and determinantal quantum Monte Carlo methods for spins, phonons and electrons, Lect. Notes Phys. 739, 277 (2008). 
[5] Z.-X. Li and H. Yao, Sign-problem-free fermionic quantum Monte Carlo: Developments and applications, Annu. Rev. Condens. Matter Phys. 10, 337 (2019).

[6] M. Marvian, D. A. Lidar, and I. Hen, On the computational complexity of curing non-stoquastic Hamiltonians, Nat. Commun. 10, 1571 (2019).

[7] J. Klassen, M. Marvian, S. Piddock, M. Ioannou, I. Hen, and B. Terhal, Hardness and ease of curing the sign problem for two-local qubit Hamiltonians, arXiv:1906.08800 (2019).

[8] S. Bravyi, D. P. Divincenzo, R. Oliveira, and B. M. Terhal, The complexity of stoquastic local Hamiltonian problems, Quantum Inf. Comput. 8, 361 (2008).

[9] M. B. Hastings, How quantum are non-negative wavefunctions? J. Math. Phys. 57, 015210 (2016).

[10] D. Hangleiter, I. Roth, D. Nagaj, and J. Eisert, Easing the Monte Carlo sign problem, Sci. Adv. 6, eabb8341 (2020).

[11] R. K. Kaul, R. G. Melko, and A. W. Sandvik, Bridging latticescale physics and continuum field theory with quantum Monte Carlo simulations, Annu. Rev. Condens. Matter Phys. 4, 179 (2013).

[12] D. M. Ceperley, Path integrals in the theory of condensed helium, Rev. Mod. Phys. 67, 279 (1995).

[13] S. Chandrasekharan and U.-J. Wiese, Meron-Cluster Solution of Fermion Sign Problems, Phys. Rev. Lett. 83, 3116 (1999).

[14] F. F. Assaad and I. F. Herbut, Pinning the Order: The Nature of Quantum Criticality in the Hubbard Model on Honeycomb Lattice, Phys. Rev. X 3, 031010 (2013).

[15] S. Chandrasekharan and A. Li, Quantum critical behavior in three dimensional lattice Gross-Neveu models, Phys. Rev. D 88, 021701(R) (2013)

[16] S. Gazit, F. F. Assaad, S. Sachdev, A. Vishwanath, and C. Wang, Confinement transition of $\mathbb{Z}_{2}$ gauge theories coupled to massless fermions: Emergent quantum chromodynamics and $s o(5)$ symmetry, Proc. Natl. Acad. Sci. USA 115, E6987 (2018).

[17] E. Berg, S. Lederer, Y. Schattner, and S. Trebst, Monte Carlo studies of quantum critical metals, Annu. Rev. Condens. Matter Phys. 10, 63 (2019)

[18] L. Wang, Y.-H. Liu, M. Iazzi, M. Troyer, and G. Harcos, Split Orthogonal Group: A Guiding Principle for Sign-ProblemFree Fermionic Simulations, Phys. Rev. Lett. 115, 250601 (2015).

[19] R. Blankenbecler, D. J. Scalapino, and R. L. Sugar, Monte Carlo calculations of coupled boson-fermion systems. I, Phys. Rev. D 24, 2278 (1981).

[20] R. R. dos Santos, Introduction to quantum Monte Carlo simulations for fermionic systems, Braz. J. Phys. 33, 36 (2003).

[21] S. Chandrasekharan, Fermion bag approach to fermion sign problems, Eur. Phys. J. A 49, 90 (2013).

[22] Z.-X. Li, Y.-F. Jiang, and H. Yao, Majorana-Time-Reversal Symmetries: A Fundamental Principle for Sign-Problem-Free Quantum Monte Carlo Simulations, Phys. Rev. Lett. 117, 267002 (2016).

[23] Z. C. Wei, C. Wu, Y. Li, S. Zhang, and T. Xiang, Majorana Positivity and the Fermion Sign Problem of Quantum Monte Carlo Simulations, Phys. Rev. Lett. 116, 250601 (2016).

[24] Z.-C. Wei, Semigroup approach to the sign problem in quantum Monte Carlo simulations, arXiv:1712.09412 (2017).

[25] C. N. Varney, C.-R. Lee, Z. J. Bai, S. Chiesa, M. Jarrell, and R. T. Scalettar, Quantum Monte Carlo study of the two- dimensional fermion Hubbard model, Phys. Rev. B 80, 075116 (2009).

[26] J. P. F. LeBlanc, A. E. Antipov, F. Becca, I. W. Bulik, G. K.-L. Chan, C.-M. Chung, Y. Deng, M. Ferrero, T. M. Henderson, C. A. Jiménez-Hoyos et al. (Simons Collaboration on the Many-Electron Problem), Solutions of the Two-Dimensional Hubbard Model: Benchmarks and Results from a Wide Range of Numerical Algorithms, Phys. Rev. X 5, 041041 (2015).

[27] A. Kantian, M. Dolfi, M. Troyer, and T. Giamarchi, Understanding repulsively mediated superconductivity of correlated electrons via massively parallel density matrix renormalization group, Phys. Rev. B 100, 075138 (2019).

[28] S. Hands, I. Montvay, S. Morrison, M. Oevers, L. Scorzato, and J. Skullerud, Numerical study of dense adjoint matter in two color QCD, Eur. Phys. J. C 17, 285 (2000).

[29] C. R. Allton, S. Ejiri, S. J. Hands, O. Kaczmarek, F. Karsch, E. Laermann, Ch. Schmidt, and L. Scorzato, QCD thermal phase transition in the presence of a small chemical potential, Phys. Rev. D 66, 074507 (2002).

[30] V. A. Goy, V. Bornyakov, D. Boyda, A. Molochkov, A. Nakamura, A. Nikolaev, and V. Zakharov, Sign problem in finite density lattice QCD, Prog. Theor. Exp. Phys. 2017, 031D01 (2017).

[31] M. Banerjee, M. Heiblum, V. Umansky, D. E. Feldman, Y. Oreg, and A. Stern, Observation of half-integer thermal Hall conductance, Nature 559, 205 (2018).

[32] C. Wang, A. Vishwanath, and B. I. Halperin, Topological order from disorder and the quantized Hall thermal metal: Possible applications to the $v=5 / 2$ state, Phys. Rev. B 98, 045112 (2018).

[33] D. F. Mross, Y. Oreg, A. Stern, G. Margalit, and M. Heiblum, Theory of Disorder-Induced Half-Integer Thermal Hall Conductance, Phys. Rev. Lett. 121, 026801 (2018).

[34] S. H. Simon, Interpretation of thermal conductance of the $v=5 / 2$ edge, Phys. Rev. B 97, 121406(R) (2018); D. E. Feldman, Comment on "interpretation of thermal conductance of the $v=5 / 2$ edge", ibid. 98, 167401 (2018).

[35] K. K. W. Ma and D. E. Feldman, Partial equilibration of integer and fractional edge channels in the thermal quantum Hall effect, Phys. Rev. B 99, 085309 (2019).

[36] L. Hu, Z. Liu, D. N. Sheng, F. D. M. Haldane, and W. Zhu, Microscopic diagnosis of universal geometric responses in fractional quantum Hall liquids, arXiv:2002.05565 (2020).

[37] M. A. Levin and X.-G. Wen, String-net condensation: A physical mechanism for topological phases, Phys. Rev. B 71, 045110 (2005).

[38] A. Smith, O. Golan, and Z. Ringel, Intrinsic sign problems in topological quantum field theories, Phys. Rev. Research 2, 033515 (2020).

[39] Z. Ringel and D. L. Kovrizhin, Quantized gravitational responses, the sign problem, and quantum complexity, Sci. Adv. 3, e1701758 (2017).

[40] H.-H. Tu, Y. Zhang, and X.-L. Qi, Momentum polarization: An entanglement measure of topological spin and chiral central charge, Phys. Rev. B 88, 195412 (2013).

[41] M. P. Zaletel, R. S. K. Mong, and F. Pollmann, Topological Characterization of Fractional Quantum Hall Ground States from Microscopic Hamiltonians, Phys. Rev. Lett. 110, 236801 (2013). 
[42] Y. J. Park and F. D. M. Haldane, Guiding-center Hall viscosity and intrinsic dipole moment along edges of incompressible fractional quantum Hall fluids, Phys. Rev. B 90, 045123 (2014).

[43] T. B. Wahl, S. T. Haßler, H.-H. Tu, J. I. Cirac, and N. Schuch, Symmetries and boundary theories for chiral projected entangled pair states, Phys. Rev. B 90, 115133 (2014).

[44] T. I. Tuegel and T. L. Hughes, Hall viscosity and momentum transport in lattice and continuum models of the integer quantum Hall effect in strong magnetic fields, Phys. Rev. B 92, 165127 (2015).

[45] J. Fröhlich and F. Gabbiani, Braid statistics in local quantum theory, Rev. Math. Phys. 02, 251 (1990).

[46] A. Kitaev, Anyons in an exactly solved model and beyond, Ann. Phys. 321, 2 (2006).

[47] D. S. Freed and M. J. Hopkins, Reflection positivity and invertible topological phases, arXiv:1604.06527 (2016).

[48] M. H. Freedman, M. Larsen, and Z. Wang, A modular functor which is universal for quantum computation, Commun. Math. Phys. 227, 605 (2002).

[49] C. Nayak, S. H. Simon, A. Stern, M. Freedman, and S. D. Sarma, Non-Abelian anyons and topological quantum computation, Rev. Mod. Phys. 80, 1083 (2008).

[50] F. Arute, K. Arya, R. Babbush, D. Bacon, J. C. Bardin, R. Barends, R. Biswas, S. Boixo, F. G. S. L. Brandao, D. A. Buell et al., Quantum supremacy using a programmable superconducting processor, Nature (London) 574, 505 (2019).

[51] C. L. Kane and M. P. A. Fisher, Quantized thermal transport in the fractional quantum Hall effect, Phys. Rev. B 55, 15832 (1997).

[52] N. Read and D. Green, Paired states of fermions in two dimensions with breaking of parity and time-reversal symmetries and the fractional quantum Hall effect, Phys. Rev. B 61, 10267 (2000).

[53] A. Cappelli, M. Huerta, and G. R. Zemba, Thermal transport in chiral conformal theories and hierarchical quantum Hall states, Nucl. Phys. B 636, 568 (2002).

[54] A. Kapustin and L. Spodyneiko, Thermal Hall conductance and a relative topological invariant of gapped two-dimensional systems, Phys. Rev. B 101, 045137 (2020).

[55] S. Jezouin, F. D. Parmentier, A. Anthore, U. Gennser, A. Cavanna, Y. Jin, and F. Pierre, Quantum limit of heat flow across a single electronic channel, Science 342, 601 (2013).

[56] M. Banerjee, M. Heiblum, A. Rosenblatt, Y. Oreg, D. E. Feldman, A. Stern, and V. Umansky, Observed quantization of anyonic heat flow, Nature (London) 545, 75 (2017).

[57] Y. Kasahara, T. Ohnishi, Y. Mizukami, O. Tanaka, S. Ma, K. Sugii, N. Kurita, H. Tanaka, J. Nasu, Y. Motome et al., Majorana quantization and half-integer thermal quantum Hall effect in a Kitaev spin liquid, Nature (London) 559, 227 (2018).

[58] A. G. Abanov and A. Gromov, Electromagnetic and gravitational responses of two-dimensional noninteracting electrons in a background magnetic field, Phys. Rev. B 90, 014435 (2014).

[59] S. Klevtsov and P. Wiegmann, Geometric Adiabatic Transport in Quantum Hall States, Phys. Rev. Lett. 115, 086801 (2015).

[60] B. Bradlyn and N. Read, Topological central charge from Berry curvature: Gravitational anomalies in trial wave func- tions for topological phases, Phys. Rev. B 91, 165306 (2015).

[61] O. Golan, C. Hoyos, and S. Moroz, Boundary central charge from bulk odd viscosity: Chiral superfluids, Phys. Rev. B 100, 104512 (2019).

[62] T. Can, Y. H. Chiu, M. Laskin, and P. Wiegmann, Emergent Conformal Symmetry and Geometric Transport Properties of Quantum Hall States on Singular Surfaces, Phys. Rev. Lett. 117, 266803 (2016).

[63] N. Schine, A. Ryou, A. Gromov, A. Sommer, and J. Simon, Synthetic Landau levels for photons, Nature (London) 534, 671 (2016).

[64] N. Schine, M. Chalupnik, T. Can, A. Gromov, and J. Simon, Electromagnetic and gravitational responses of photonic Landau levels, Nature 565, 173 (2019).

[65] R. Bhattacharyya, M. Banerjee, M. Heiblum, D. Mahalu, and V. Umansky, Melting of Interference in the Fractional Quantum Hall Effect: Appearance of Neutral Modes, Phys. Rev. Lett. 122, 246801 (2019).

[66] B. Rosenow, I. P. Levkivskyi, and B. I. Halperin, Current Correlations from a Mesoscopic Anyon Collider, Phys. Rev. Lett. 116, 156802 (2016).

[67] H. Bartolomei, M. Kumar, R. Bisognin, A. Marguerite, J.-M. Berroir, E. Bocquillon, B. Plaçais, A. Cavanna, Q. Dong, U. Gennser et al., Fractional statistics in anyon collisions, Science 368, 173 (2020).

[68] See Supplemental Material at http://link.aps.org/supplemental/ 10.1103/PhysRevResearch.2.043032 for a Mathematica notebook containing computations used to produce Table I and Fig. 8.

[69] P. H. Bonderson, Non-Abelian anyons and interferometry, Ph.D. thesis, California Institute of Technology (2007).

[70] T. Lan, L. Kong, and X.-G. Wen, Theory of (2+1)-dimensional fermionic topological orders and fermionic/bosonic topological orders with symmetries, Phys. Rev. B 94, 155113 (2016).

[71] P.-S. Hsin, Y.-H. Lin, N. M. Paquette, and J. Wang, An effective field theory for fractional quantum Hall systems near $v=5 / 2$, arXiv:2005.10826 (2020).

[72] J. Wang, X.-G. Wen, and E. Witten, Symmetric Gapped Interfaces of SPT and Set States: Systematic Constructions, Phys. Rev. X 8, 031048 (2018).

[73] B. Zeng, X. Chen, D.-L. Zhou, and X.-G. Wen, Quantum Information Meets Quantum Matter (Springer, Berlin, 2019).

[74] X. Chen, Z.-C. Gu, Z.-X. Liu, and X.-G. Wen, Symmetry protected topological orders and the group cohomology of their symmetry group, Phys. Rev. B 87, 155114 (2013).

[75] A. Kapustin, R. Thorngren, A. Turzillo, and Z. Wang, Fermionic symmetry protected topological phases and cobordisms, J. High Energy Phys. 12 (2015) 052.

[76] A. Kapustin and R. Thorngren, Fermionic SPT phases in higher dimensions and bosonization, J. High Energy Phys. 10 (2017) 80 .

[77] X.-L. Qi and S.-C. Zhang, Topological insulators and superconductors, Rev. Mod. Phys. 83, 1057 (2011).

[78] X. G. Wen, Topological orders in rigid states, Int. J. Mod. Phys. B 04, 239 (1990).

[79] X. Chen, Z.-C. Gu, and X.-G. Wen, Local unitary transformation, long-range quantum entanglement, wave function renormalization, and topological order, Phys. Rev. B 82, 155138 (2010). 
[80] L. Cincio and G. Vidal, Characterizing Topological Order by Studying the Ground States on an Infinite Cylinder, Phys. Rev. Lett. 110, 067208 (2013).

[81] L. Savary and L. Balents, Quantum spin liquids: A review, Rep. Prog. Phys. 80, 016502 (2016).

[82] M. Levin and A. Stern, Fractional Topological Insulators, Phys. Rev. Lett. 103, 196803 (2009).

[83] A. Stern, Fractional topological insulators: A pedagogical review, Annu. Rev. Condens. Matter Phys. 7, 349 (2016).

[84] P. Ginsparg, Applied conformal field theory, arXiv:hep-th/ 9108028 (1988).

[85] P. Francesco, P. Mathieu, and D. Sénéchal, Conformal Field Theory, Graduate Texts in Contemporary Physics (Island Press, New York, 1996).

[86] F. D. M. Haldane, Model for a Quantum Hall Effect Without Landau Levels: Condensed-Matter Realization of the "Parity Anomaly", Phys. Rev. Lett. 61, 2015 (1988).

[87] X.-L. Qi, T. L. Hughes, and S.-C. Zhang, Topological field theory of time-reversal invariant insulators, Phys. Rev. B 78, 195424 (2008).

[88] S. Ryu, A. P. Schnyder, A. Furusaki, and A. W. W. Ludwig, Topological insulators and superconductors: Tenfold way and dimensional hierarchy, New J. Phys. 12, 065010 (2010).

[89] H. Takagi, T. Takayama, G. Jackeli, G. Khaliullin, and S. E. Nagler, Concept and realization of Kitaev quantum spin liquids, Nat. Rev. Phys. 1, 264 (2019).

[90] E. Witten, Quantum field theory and the Jones polynomial, Commun. Math. Phys. 121, 351 (1989).

[91] A. Gromov, G. Y. Cho, Y. You, A. G. Abanov, and E. Fradkin, Framing Anomaly in the Effective Theory of the Fractional Quantum Hall Effect, Phys. Rev. Lett. 114, 016805 (2015).

[92] A. Gromov, K. Jensen, and A. G. Abanov, Boundary Effective Action for Quantum Hall States, Phys. Rev. Lett. 116, 126802 (2016).

[93] O. Golan and A. Stern, Probing topological superconductors with emergent gravity, Phys. Rev. B 98, 064503 (2018).

[94] H. Moradi and X.-G. Wen, Universal Wave-Function Overlap and Universal Topological Data from Generic Gapped Ground States, Phys. Rev. Lett. 115, 036802 (2015).

[95] A. Altland and B. D. Simons, Condensed Matter Field Theory (Cambridge University Press, Cambridge, 2010).

[96] J. Yu, X.-H. Zhang, and S.-P. Kou, Majorana edge states for $\mathbb{Z}_{2}$ topological orders of the Wen plaquette and toric code models, Phys. Rev. B 87, 184402 (2013).

[97] S. Lloyd, Universal quantum simulators, Science 273, 1073 (1996).

[98] J.-W. Mei and X.-G. Wen, Modular matrices from universal wave-function overlaps in Gutzwiller-projected parton wave functions, Phys. Rev. B 91, 125123 (2015).

[99] G. E. Volovik, The Universe in a Helium Droplet (Oxford University Press, New York, 2009).

[100] F. Rose, O. Golan, and S. Moroz, Hall viscosity and conductivity of two-dimensional chiral superconductors, SciPost Phys. 9, 006 (2020).

[101] S. Sachdev, Quantum Phase Transitions, 2nd ed. (Cambridge University Press, Cambridge, 2011).

[102] S. M. A. Rombouts, K. Heyde, and N. Jachowicz, Quantum Monte Carlo Method for Fermions, Free of Discretization Errors, Phys. Rev. Lett. 82, 4155 (1999).
[103] C. Wu and S.-C. Zhang, Sufficient condition for absence of the sign problem in the fermionic quantum Monte Carlo algorithm, Phys. Rev. B 71, 155115 (2005).

[104] J. S. Hofmann, F. F. Assaad, R. Queiroz, and E. Khalaf, Search for correlation-induced adiabatic paths between distinct topological insulators, Phys. Rev. Research 2, 023390 (2020).

[105] J. Nasu, J. Yoshitake, and Y. Motome, Thermal Transport in the Kitaev Model, Phys. Rev. Lett. 119, 127204 (2017).

[106] Z.-C. Gu, Z. Wang, and X.-G. Wen, Classification of two-dimensional fermionic and bosonic topological orders, Phys. Rev. B 91, 125149 (2015).

[107] M. Nakahara, Geometry, Topology and Physics (CRC Press, Boca Raton, FL, 2003).

[108] Y. Zhang, T. Grover, A. Turner, M. Oshikawa, and A. Vishwanath, Quasiparticle statistics and braiding from groundstate entanglement, Phys. Rev. B 85, 235151 (2012).

[109] H. Moradi and X.-G. Wen, Universal topological data for gapped quantum liquids in three dimensions and fusion algebra for non-Abelian string excitations, Phys. Rev. B 91, 075114 (2015).

[110] H. He, H. Moradi, and X.-G. Wen, Modular matrices as topological order parameter by a gauge-symmetry-preserved tensor renormalization approach, Phys. Rev. B 90, 205114 (2014).

[111] J.-W. Mei, J.-Y. Chen, H. He, and X.-G. Wen, Gapped spin liquid with $\mathbb{Z}_{2}$ topological order for the kagome Heisenberg model, Phys. Rev. B 95, 235107 (2017).

[112] Q. Zhang, Z.-Q. Wang, and G.-M. Zhang, Non-Hermitian effects of the intrinsic signs in topologically ordered wavefunctions, arXiv:2005.03841 (2020).

[113] T. Ma, Z. Huang, F. Hu, and H.-Q. Lin, Pairing in graphene: A quantum Monte Carlo study, Phys. Rev. B 84, 121410(R) (2011).

[114] A. M. Black-Schaffer and C. Honerkamp, Chirald-wave superconductivity in doped graphene, J. Phys.: Condens. Matter 26, 423201 (2014).

[115] C.-H. Lin and M. Levin, Generalizations and limitations of string-net models, Phys. Rev. B 89, 195130 (2014).

[116] A. C. Potter and A. Vishwanath, Protection of topological order by symmetry and many-body localization, arXiv:1506.00592 (2015).

[117] N. Tantivasadakarn and A. Vishwanath, Full commuting projector Hamiltonians of interacting symmetry-protected topological phases of fermions, Phys. Rev. B 98, 165104 (2018).

[118] T. B. Wahl, H.-H. Tu, N. Schuch, and J. I. Cirac, Projected Entangled-Pair States can Describe Chiral Topological States, Phys. Rev. Lett. 111, 236805 (2013).

[119] J. Dubail and N. Read, Tensor network trial states for chiral topological phases in two dimensions and a no-go theorem in any dimension, Phys. Rev. B 92, 205307 (2015).

[120] J.-Y. Chen, L. Vanderstraeten, S. Capponi, and D. Poilblanc, Non-Abelian chiral spin liquid in a quantum antiferromagnet revealed by an iPEPS study, Phys. Rev. B 98, 184409 (2018).

[121] J. H. Son and J. Alicea, Commuting-projector Hamiltonians for chiral topological phases built from parafermions, Phys. Rev. B 97, 245144 (2018).

[122] G. Torlai, J. Carrasquilla, M. T. Fishman, R. G. Melko, and M. P. A. Fisher, Wavefunction positivization via automatic differentiation, Phys. Rev. Research 2, 032060(R) (2020). 
[123] A. Alexandru, P. F. Bedaque, H. Lamm, and S. Lawrence, Finite-density Monte Carlo calculations on sign-optimized manifolds, Phys. Rev. D 97, 094510 (2018).

[124] A. Alexandru, G. Basar, P. F. Bedaque, and N. C. Warrington, Complex paths around the sign problem, arXiv:2007.05436 (2020).

[125] H. J. M. van Bemmel, D. F. B. ten Haaf, W. van Saarloos, J. M. J. van Leeuwen, and G. An, Fixed-Node Quantum Monte Carlo Method for Lattice Fermions, Phys. Rev. Lett. 72, 2442 (1994).

[126] S. Zhang, J. Carlson, and J. E. Gubernatis, Constrained path Monte Carlo method for fermion ground states, Phys. Rev. B 55, 7464 (1997).

[127] P. Broecker, J. Carrasquilla, R. G. Melko, and S. Trebst, Machine learning quantum phases of matter beyond the fermion sign problem, Sci. Rep. 7, 8823 (2017).

[128] R. Rossi, Determinant Diagrammatic Monte Carlo Algorithm in the Thermodynamic Limit, Phys. Rev. Lett. 119, 045701 (2017).

[129] M. P. Zaletel, Detecting two-dimensional symmetry-protected topological order in a ground-state wave function, Phys. Rev. B 90, 235113 (2014).

[130] E. Witten, Fermion path integrals and topological phases, Rev. Mod. Phys. 88, 035001 (2016).

[131] K. Shiozaki, H. Shapourian, and S. Ryu, Many-body topological invariants in fermionic symmetry-protected topological phases: Cases of point group symmetries, Phys. Rev. B 95, 205139 (2017).

[132] K. Shiozaki, H. Shapourian, K. Gomi, and S. Ryu, Manybody topological invariants for fermionic short-range entangled topological phases protected by antiunitary symmetries, Phys. Rev. B 98, 035151 (2018).

[133] R. Bondesan and Z. Ringel, Classical topological paramagnetism, Phys. Rev. B 95, 174418 (2017).

[134] S. D. Geraedts and O. I. Motrunich, Monte Carlo study of a $u(1) \times u(1)$ system with $\pi$-statistical interaction, Phys. Rev. B 85, 045114 (2012).

[135] S. D. Geraedts and O. I. Motrunich, Phases and phase transitions in a $u(1) \times u(1)$ system with $\theta=2 \pi / 3$ mutual statistics, Phys. Rev. B 86, 045106 (2012).

[136] S. Gazit and A. Vishwanath, Bosonic topological phase in a paired superfluid, Phys. Rev. B 93, 115146 (2016).

[137] T. Lan, L. Kong, and X.-G. Wen, Classification of (2+1)dimensional topological order and symmetry-protected topological order for bosonic and fermionic systems with on-site symmetries, Phys. Rev. B 95, 235140 (2017).

[138] D. Aasen, E. Lake, and K. Walker, Fermion condensation and super pivotal categories, J. Math. Phys. 60, 121901 (2019).

[139] A.Yu. Kitaev, Fault-tolerant quantum computation by anyons, Ann. Phys. 303, 2 (2003).

[140] M. Fremling, T. H. Hansson, and J. Suorsa, Hall viscosity of hierarchical quantum Hall states, Phys. Rev. B 89, 125303 (2014). 